\title{
HECTOSPEC AND HYDRA SPECTRA OF INFRARED LUMINOUS SOURCES IN THE AKARI NORTH ECLIPTIC POLE SURVEY FIELD
}

\author{
Hyunjin Shim ${ }^{1,2,3,7}$, Myungshin Im ${ }^{2,3,7}$, Jongwan Ko ${ }^{4,7}$, Yiseul JeON ${ }^{2,3}$, Marios Karouzos ${ }^{3}$, \\ Seong Jin Kim ${ }^{2}$, Hyung Mok LeE ${ }^{2}$, Casey Papovich ${ }^{5}$, Christopher Willmer ${ }^{6}$, And Benjamin J. WeineR ${ }^{6}$ \\ ${ }^{1}$ Department of Earth Science Education, Kyungpook National University, Daegu 702-701, Korea; hjshim@knu.ac.kr \\ ${ }^{2}$ Department of Physics \& Astronomy, FPRD, Seoul National University, Seoul 151-742, Korea \\ ${ }^{3}$ Center for the Exploration of the Origin of the Universe (CEOU), Seoul National University, Seoul 151-742, Korea \\ ${ }^{4}$ Korea Astronomy and Space Science Institute (KASI), Daejeon 305-348, Korea \\ ${ }^{5}$ George P. and Cynthia W. Mitchell Institute for Fundamental Physics and Astronomy, Department of Physics, \\ Texas A\&M University, College Station, TX 77843, USA \\ ${ }^{6}$ Steward Observatory, University of Arizona, 933 North Cherry Avenue, Tucson, AZ 85721, USA \\ Received 2013 February 14; accepted 2013 June 24; published 2013 August 2
}

\begin{abstract}
We present spectra of 1796 sources selected in the AKARI North Ecliptic Pole Wide Survey field, obtained with MMT/Hectospec and WIYN/Hydra, for which we measure 1645 redshifts. We complemented the generic fluxlimited spectroscopic surveys at $11 \mu \mathrm{m}$ and $15 \mu \mathrm{m}$, with additional sources selected based on the MIR and optical colors. In MMT/Hectospec observations, the redshift identification rates are $\sim 80 \%$ for objects with $R<21.5 \mathrm{mag}$. On the other hand, in WIYN/Hydra observations, the redshift identification rates are $\sim 80 \%$ at $R$ magnitudes brighter than 19 mag. The observed spectra were classified through the visual inspection or from the line diagnostics. We identified 1128 star-forming or absorption-line-dominated galaxies, 198 Type-1 active galactic nuclei (AGNs), 8 Type-2 AGNs, 121 Galactic stars, and 190 spectra in unknown category due to low signal-to-noise ratio. The spectra were flux-calibrated but to an accuracy of $0.1-0.18$ dex for most of the targets and worse for the remainder. We derive star formation rates (SFRs) from the mid-infrared fluxes or from the optical emission lines, showing that our sample spans an SFR range of 0.1 to a few hundred $M_{\odot} \mathrm{yr}^{-1}$. We find that the extinction inferred from the difference between the IR and optical SFR increases as the IR luminosity increases but with a large scatter.
\end{abstract}

Key words: catalogs - galaxies: distances and redshifts - infrared: galaxies - surveys - techniques: spectroscopic

Online-only material: color figures, machine-readable tables

\section{INTRODUCTION}

The infrared space telescope AKARI (Murakami et al. 2007) successfully carried out its missions during its lifetime between 2006 February and 2009 February. Before the end of the cold phase in 2007 August, in addition to the all-sky surveys at midand far-infrared wavelengths (Ishihara et al. 2010; Doi et al. 2012), AKARI conducted a survey covering the North Ecliptic Pole (NEP; R.A. $=17^{\mathrm{h}} 55^{\mathrm{m}} 24^{\mathrm{s}}$, Decl. $\left.=+66^{\circ} 37^{\prime} 32^{\prime \prime}\right)$ over which the visibility is high for the polar Sun synchronous orbit of AKARI.

The NEP survey is composed of two programs, the Deep (NEP-Deep) and Wide (NEP-Wide) surveys (Matsuhara et al. 2006). The NEP-Wide covers a $5.4 \mathrm{deg}^{2}$ field with relatively short exposure times (90-150 s; Lee et al. 2009; Kim et al. 2012), while the NEP-Deep covers a $0.38 \mathrm{deg}^{2}$ area inside the NEP-Wide field with exposure 3-10 times longer. Both the Deep and the Wide surveys are done using the comprehensive filter sets of the InfraRed Camera (IRC; Onaka et al. 2007) covering 2-24 $\mu \mathrm{m}$. The filter sets used are $N 2, N 3, N 4, S 7, S 9 W, S 11, L 15, L 18 W$, and $L 24$, while the number next to each alphabet indicates the central wavelength of the filter in $\mu \mathrm{m}$. The width of the $S 9 \mathrm{~W}$ and $L 18 \mathrm{~W}$ filters is wider than that of other filters. The continuous wavelength coverage of IRC enables detailed study of the MIR spectral energy distribution (SED) of galaxies.

\footnotetext{
7 Guest Observer, Kitt Peak National Observatory, National Optical Astronomy Observatory, which is operated by the Association of Universities for Research in Astronomy (AURA) under cooperative agreement with the National Science Foundation.
}

A noticeable aspect of the AKARI NEP-Wide survey, in addition to its wide area coverage and continuous filter coverage, is that a large number of ground-based ancillary data sets are also available for this field, supporting the identification and study of the infrared sources. Specifically, the survey is complemented by deep optical images with MegaCam at the Canada-France-Hawaii Telescope (CFHT; Hwang et al. 2007) and Seoul National University $4 \mathrm{k} \times 4 \mathrm{k}$ Camera (SNUCAM) on the $1.5 \mathrm{~m}$ Richey-Chretian, AZT-22 telescope at the Maidanak Observatory (Im et al. 2010; Jeon et al. 2010), as well as deep NIR images by FLAMINGOS at KPNO (Y. Jeon et al., in preparation). Published or ongoing science with this survey field includes study of MIR luminosity function (Goto et al. 2010), nature of MIR-bright or polycyclic aromatic hydrocarbon (PAH)-luminous sources (Takagi et al. 2010), and properties of galaxies in NEP supercluster (Ko et al. 2012).

While the photometric data alone allows studying a whole range of topics investigating galaxy properties in infrared wavelengths, an important limitation is the lack of spectroscopic surveys covering the entire survey field. For example, the construction of luminosity function based on the photometric redshifts is highly uncertain especially when the accuracy of photometric redshift is ambiguous due to the existence of many strong emission lines. Although multi-wavelength imaging is a good survey strategy as a starting point, spectroscopic observations are in the end necessary in order to investigate the nature of extragalactic sources, including star formation rates (SFRs), chemical properties, and luminosity functions. Under such motivations, we carried out an extensive spectroscopic survey over the AKARI NEP-Wide survey field using the MMT/Hectospec and WIYN/ 
Table 1

Spectroscopic Target Selection

\begin{tabular}{|c|c|c|c|}
\hline & Category & $N_{\text {source }}$ & Criteria \\
\hline & Primary & 1155 & $S 11<18.5 \mathrm{mag}$ and $L 15<17.9 \mathrm{mag}$ \\
\hline \multirow{9}{*}{ Secondary } & High- $z$ candidates & 52 & $\begin{array}{l}g-r>1.5 \text { and } g-r>2.5 \times(r-i)+0.3, \\
B-R>2 \text { and } B-R>4 \times(R-I)+0.8\end{array}$ \\
\hline & AGN candidates & 192 & $N 2-N 4>0$ and $S 7-S 11>0$ \\
\hline & Supercluster candidates & 158 & Ko et al. (2012) \\
\hline & radio sources $^{\mathrm{a}}$ & 75 & White et al. (2010) \\
\hline & $z \sim 1$ cluster candidates & 4 & Goto et al. (2008) \\
\hline & BzK galaxies & 6 & Hanami et al. (2012) \\
\hline & FIR sources ${ }^{\mathrm{a}}$ & 52 & Oyabu et al. (2011) \\
\hline & PAH-luminous galaxies ${ }^{\mathrm{a}}$ & 102 & Ohyama et al. (2009), Takagi et al. (2010) \\
\hline & Total & 1796 & \\
\hline
\end{tabular}

Note. ${ }^{a}$ Targets selected with these criteria largely overlap with the primary sample. These were given higher priority when the spectroscopic masks were designed.

Hydra multi-fiber spectrographs. Spectroscopic follow-ups of Spitzer wide-field (a few deg $^{2}$ or more) IR surveys already exist (e.g., Marleau et al. 2007; Papovich et al. 2006; Owen \& Morrison 2009). However, this survey adds a unique aspect by selecting main targets based on the $11 \mu \mathrm{m}$ and $15 \mu \mathrm{m}$ fluxes (not covered by Spitzer), which allow us to perform an analysis of the evolution of the "contiguous" IR SED shape of distant galaxies at $z<1$ from NIR to MIR.

This paper is organized as follows: in Section 2, we describe how the follow-up spectroscopic observations were designed, conducted, and finally how the obtained data were reduced. In Section 3, we present the redshift catalog as the first result. The redshift distribution of the infrared sources is discussed, in addition to the survey completeness as a function of optical magnitude. In Sections 4 and 5, we present the line flux measurements for significant emission lines, and discuss the relation between the SFRs estimated from the optical emission lines and from the infrared luminosity. Emission line diagnostics for narrow-line active galactic nuclei (AGNs) classification is also presented. Throughout this paper, we use a cosmology with $\Omega_{M}=0.3, \Omega_{\Lambda}=0.7$, and $H_{0}=70 \mathrm{~km} \mathrm{~s}^{-1} \mathrm{Mpc}^{-1}$. All magnitudes mentioned are in the $\mathrm{AB}$ magnitude system (Oke 1974).

\section{OBSERVATIONS AND DATA REDUCTION}

\subsection{Spectroscopic Target Selection}

Targets for the spectroscopic observation were selected from the optical to mid-infrared band-merged photometry catalog over the NEP-Wide field (Kim et al. 2012). In this catalog, the AKARI IR sources are matched within different AKARI bands and then matched to entries in the optical catalogs (CFHT ugriz, Maidanak BRI; Hwang et al. 2007; Jeon et al. 2010) by identifying the nearest object within a matching radius of $4^{\prime \prime}$. In addition to the band-merged catalog presented in Kim et al. (2012), we also prepared a catalog of objects with optical detection but without AKARI detection, in order to select supplementary targets based on the optical colors. The construction of this catalog is done similarly to the AKARI bandmerged catalog as described above. The stellarity information from the optical band data, as well as a NIR color $(N 2-N 4$; Lee et al. 2007), were used to exclude Galactic stars, where we identify stars using the stellarity cuts greater than 0.9 and a NIR color $N 2-N 4$ less than -1 .

Table 1 summarizes the target selection criteria. The primary targets for spectroscopy were MIR sources in flux-limited samples at $11 \mu \mathrm{m}\left(f_{S 11}>150 \mu \mathrm{Jy}\right)$ and at $15 \mu \mathrm{m}\left(f_{L 15}>250 \mu \mathrm{Jy}\right)$, i.e., corresponding to magnitudes brighter than $18.5 \mathrm{mag}$ and $17.9 \mathrm{mag}$, respectively. These flux limits are roughly consistent with the 50\% completeness limit of the NEP-Wide field images in the corresponding filters (Kim et al. 2012). We chose sources with fluxes higher than the flux cuts in both $S 11$ and $L 15$ bands. An additional $R$-band magnitude cut is imposed on these MIR flux-limited samples, in order to select objects that are sufficiently bright to yield spectra with reasonable signal-to-noise ratio $(\mathrm{S} / \mathrm{N})$. The $R$-band cuts are $16 \mathrm{mag}<R<22.5 \mathrm{mag}$ for Hectospec, and $16 \mathrm{mag}<R<21 \mathrm{mag}$ for Hydra observations, due to the differences of the two instrument-telescope combinations in depths that they can reach. The magnitude cut at the bright end is introduced in order to avoid saturation in signal. The objects satisfying the primary target selection cut are randomly chosen, to make the sample statistically unbiased. There are roughly $\sim 850$ objects in a circular area are with one degree diameter (matches the field of view of Hectospec and Hydra) satisfying the above selection criteria. This is 3-12 times larger than the number of fibers available in a single setup ( 3 for Hectospec and 12 for Hydra), so we are sampling only a subset of the objects ( $8 \%-33 \%$ for the Hydra and Hectospec observations respectively) in the MIR-flux/R-band limited sample.

Besides the targets selected from the MIR-flux/ $R$-band limited selection, a much smaller number of special targets were selected in order to study various categories of sources of specific scientific interests. Since the number of special targets is small compared to the number of primary targets, a higher priority was given to the special targets when assigning fibers for each target during the setup of the spectroscopic observations (see the observations section below; Sections 2.2.1, and 2.3.1). These special targets include objects such as high-redshift galaxy candidates, AGN candidates (Lee et al. 2007), NEP supercluster member candidates (Ko et al. 2012; Shim et al. 2011), radio sources (White et al. 2010), $z \sim 1$ cluster galaxy candidates (Goto et al. 2008), BzK galaxies (Hanami et al. 2012), far-infrared sources (Oyabu et al. 2011), and PAH-luminous galaxies (Ohyama et al. 2009; Takagi et al. 2010). 

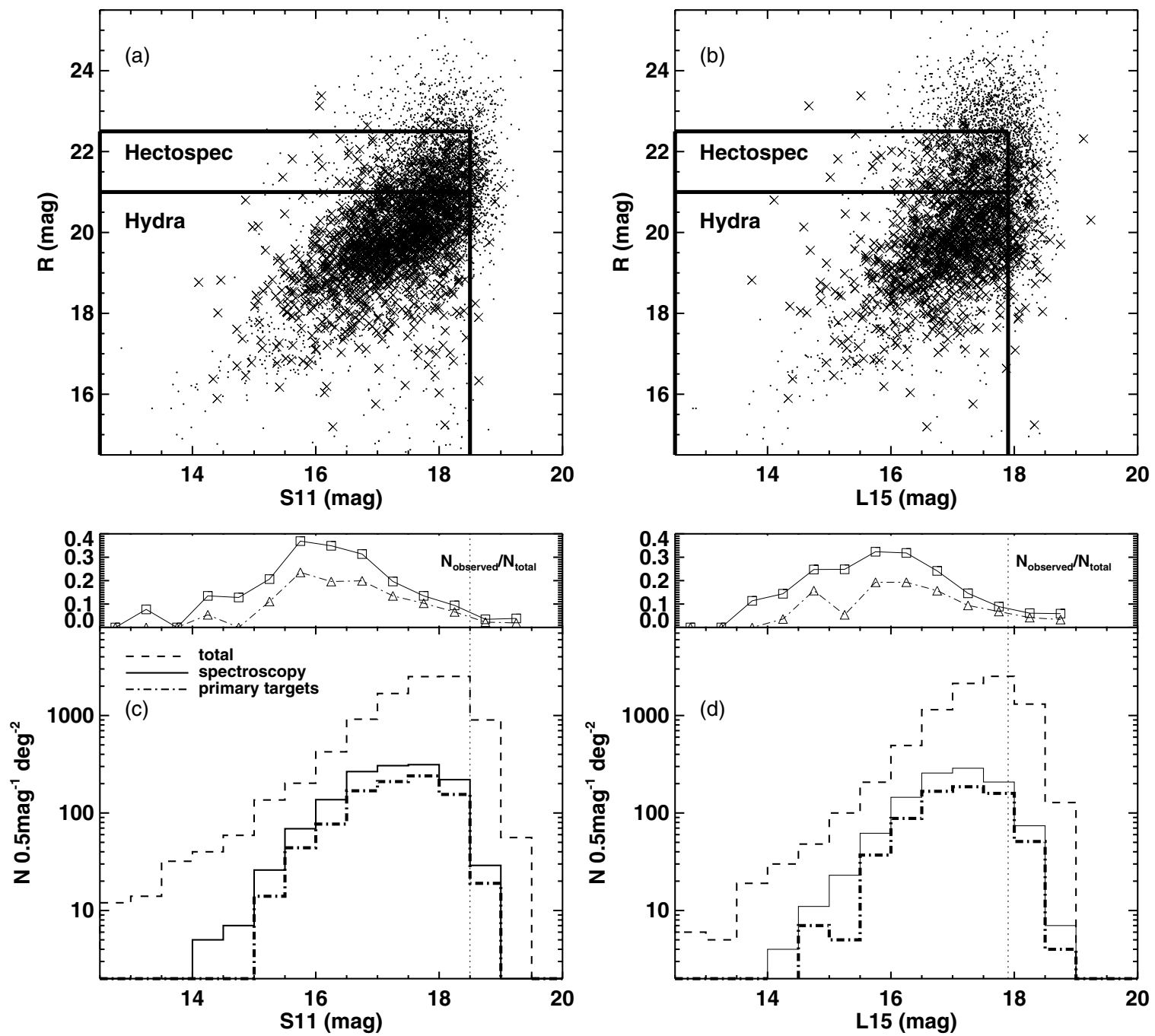

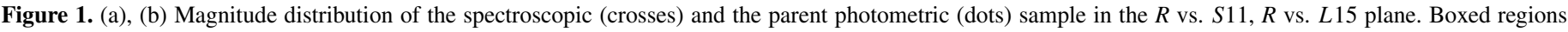

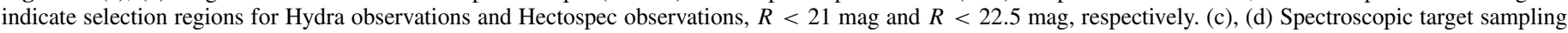

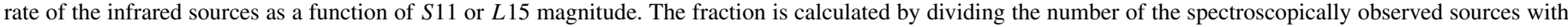

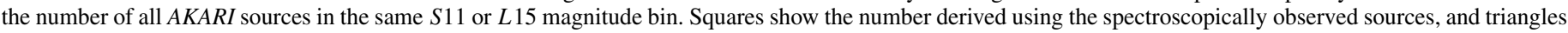

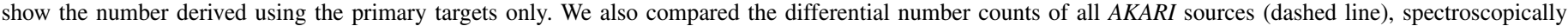
targeted sources (solid line), and spectroscopically observed primary targets (dot-dashed line) in the $11 \mu \mathrm{m}$ and $15 \mu \mathrm{m}$.

A detailed description on how the secondary sample is constructed is as follows. The high-redshift galaxy candidates are selected with typical drop-out criteria of $u-g>1.4$, $g-r>1.5, B-R>2$, and colors being blue in the redder color indices either in the $u-g$ versus $g-r$ color-color plane, $g-r$ versus $r-i$ plane, or $B-R$ versus $R-I$ plane. These criteria intend to select galaxies at $z \sim 3$ (u-dropout) and at $z \sim 4$ ( $g$ dropouts or $B$-dropouts) ${ }^{8}$ AGN candidates are selected based on the $N 2-N 4, S 7-S 11$ colors (Lee et al. 2007; N2-N4 $>0$, $S 7-S 11>0$ ), which reflect the power-law SED shape of an AGN that monotonically increases as a function of wavelength in the MIR range. Many of these AGNs satisfy the main target selection criteria, therefore choosing AGN candidates and giving them a higher priority in the fiber assignment mean that we give high weight for spectroscopic target selection of objects with $N 2-N 4>0$ and $S 7-S 11>0$. Member candidates of a known supercluster in the NEP region are selected based on the red sequence at $z=0.087$ (Shim et al. 2011), applying a color cut of $-0.7<N 3-N 4<-0.4$. For other targets,

\footnotetext{
8 Ten of these objects are not detected in any of the AKARI bands.
}

(radio sources with $f_{1.4 \mathrm{GHz}}>5 \mathrm{mJy}$, cluster galaxy candidates at $0.9<z<1.3$, BzK galaxies, far-infrared sources, and PAH luminous galaxies), please refer to the references. The same $R$-band magnitude cut as the primary sample was applied to all of these secondary target selection as well.

Figure 1 illustrates the fraction of sources spectroscopically observed among the entire AKARI infrared sources in the $R$ versus $S 11$ magnitude-magnitude plane, and in the $R$ versus $L 15$ plane. In panels (a) and (b) of Figure 1, crosses represent spectroscopically observed sources while dots represent all infrared sources detected either in $S 11$ (a) or in $L 15$ (b). Here, the spectroscopically observed sources include both the primary targets and the secondary targets. At $f_{S 11}>150 \mu$ Jy (i.e., $S 11<18.5 \mathrm{mag}$ ), $62 \%$ of the entire infrared sources satisfy the $R<21$ magnitude cut and $78 \%$ satisfy the $R<22.5$ magnitude cut. We also compared the differential number count of sources as a function of $11 \mu \mathrm{m}$ and $15 \mu \mathrm{m}$ magnitudes between those spectroscopically observed and all infrared sources (panels (c) and (d) of Figure 1). The dashed line shows the differential number count of all $S 11$ or $L 15$ sources, the solid line represents the number count of spectroscopically observed sources, and 

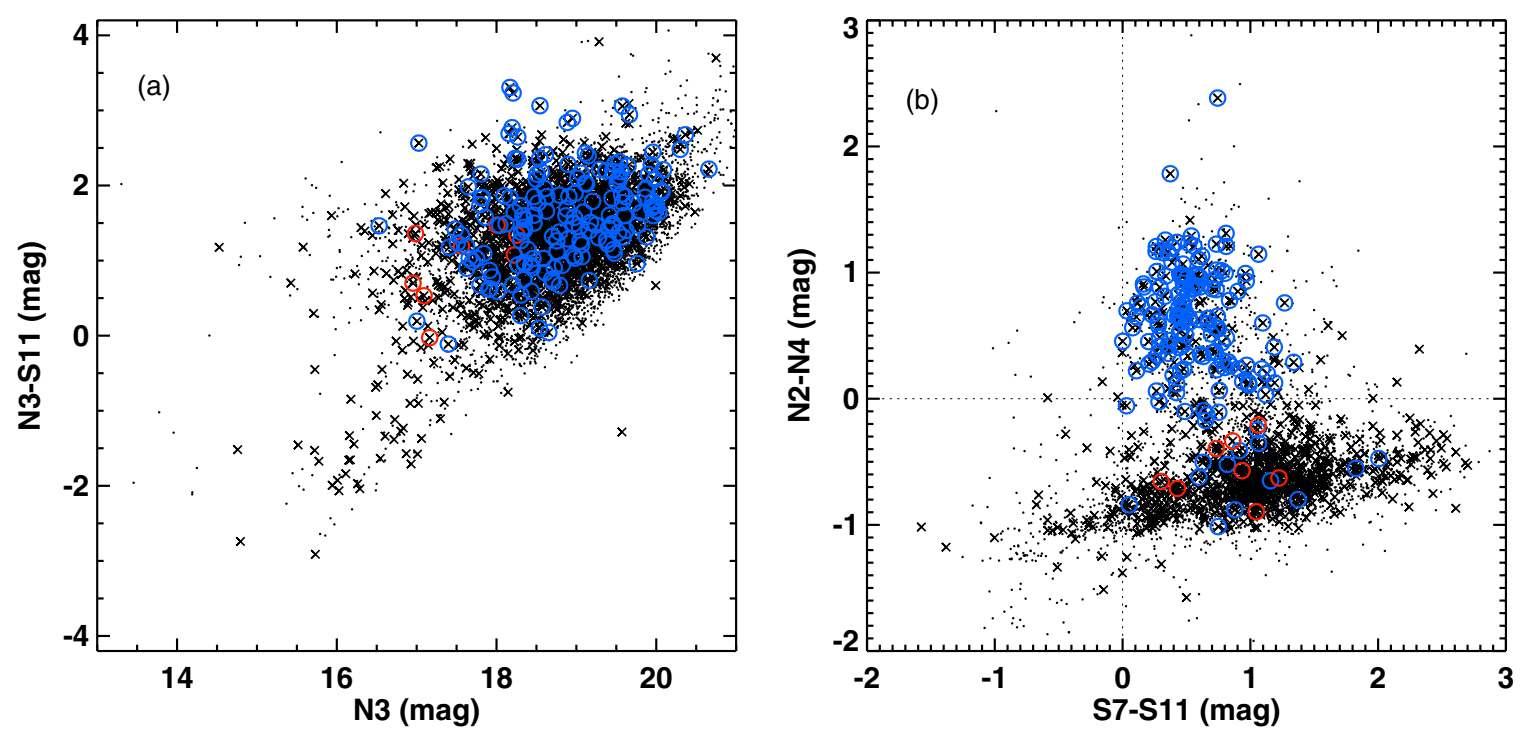

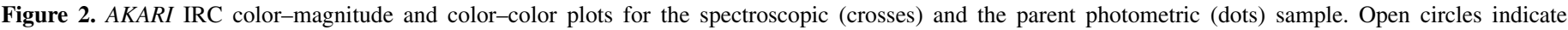

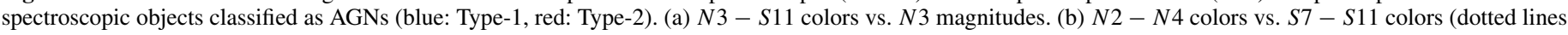

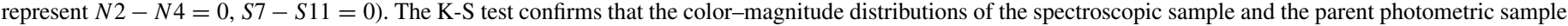
are $>99 \%$ identical

(A color version of this figure is available in the online journal.)

the dot-dashed line illustrates the number count of primary targets only. The fraction of the spectroscopically observed sources (both primary and secondary) reaches as high as $\sim 40 \%$ at $11 \mu \mathrm{m}$ flux of $1 \mathrm{mJy}$. However, there exists a trend that spectroscopic follow-up rate decreases at a fainter infrared flux level. This trend should be considered when the data are used in statistical studies, including the derivation of the infrared luminosity function.

Lastly, to check whether the selected infrared sources are robust representatives of $A K A R I$ sources, we compared the infrared color-magnitude plot of all sources in the NEP-Wide field with that of spectroscopically targeted sources. Figure 2 shows the distribution of galaxies selected for the spectroscopic observations (crosses) with the parent photometric sample (dots) for different MIR color-magnitude and color-color planes. Panel (a) shows the $N 3-S 11$ versus $N 3$, and panel (b) shows the $N 2-N 4$ versus $S 7-S 11$. Especially in the color-color plane, the locations of the sources spectroscopically classified as AGNs (circles; for optical spectral classification, please refer to Section 3.1) are clearly distinguished, suggesting that AKARI colors are efficient tools to select AGNs. According to the Kolmogorov-Smirnov test, the spectroscopic and the parent photometric samples are identical at a probability level higher than $99 \%$.

\subsection{MMT/Hectospec}

\subsubsection{Observations}

Hectospec is a 300 fiber fed spectrograph mounted on the MMT (Fabricant et al. 2008). Each fiber covers a 1".5 aperture, and the observations used the 270 line $\mathrm{mm}^{-1}$ grating covering $\sim 3700 \AA$ to $\sim 8500 \AA$, with a spectral resolution of about $6.2 \AA$ and dispersion of $1.2 \AA$ pixel $^{-1}$. The observations were executed in queue mode: a total of five configurations were observed between 2008 May and November, with each configuration covering an area within a 1 deg diameter circle. The central coordinates, the total on-source exposure time, and the observational dates are summarized in Table 2. The total exposure time per field was $\sim 1 \mathrm{hr}$, divided into five integrations in order to reduce the effect of cosmic ray hits.

We generated Hectospec configurations using the XFITFIBS ${ }^{9}$ software. In addition to the sources for spectroscopic followup, we included fibers for spectro-photometric standard stars and blank skies for flux calibration and sky line subtraction. In every configuration, we placed four to six F-type stars as spectro-photometric standards. The selection criteria for F-type stars are $16.5<r<18.5$ and $0.1 \leqslant(g-r) \leqslant 0.3$ when selected from the Hwang et al. (2007) catalog, $15<R<18$, $0.4 \leqslant(B-R) \leqslant 0.8$, and $0.15 \leqslant(R-I) \leqslant 0.25$ when selected from the Jeon et al. (2010) catalog. Typically $\sim 250$ fibers per setup are assigned to the targets. Secondary targets were given the higher priority in the fiber assignment than the primary targets due to their small number. After assigning targets, the remaining 30-40 fibers were placed on random blank-sky positions for the effective sky subtraction.

Figure 3(a) shows the locations of Hectospec configurations in relation to the photometric surveys with the $A K A R I$ NEP-Wide in the MIR and CFHT (Hwang et al. 2007) and Maidanak (Jeon et al. 2010) in the optical.

\subsubsection{Data Reduction}

In order to reduce the Hectospec data, we used the HSRED package written by R. Cool, ${ }^{10}$ specifically developed for the reduction of data from Hectospec (Kochanek et al. 2012). The package is based on the IDL pipeline developed for the reduction of Sloan Digital Sky Survey (SDSS) spectra. After cosmic ray removal from the two-dimensional images using the IDL version of L.A.cosmic (van Dokkum 2001), HSRED performs the standard reduction by correcting for bias, flat fields, fringes, and illumination (if twilight flats were taken), performing wavelength calibration (from HeNeAr lamps) and sky subtraction, and finally, extracting one-dimensional (1D) spectra. The extracted 1D spectra are flux-calibrated using the simultaneously observed F-type stars. The observed F-type star

\footnotetext{
9 http://www.cfa.harvard.edu/ john/xfitfibs/

$10 \mathrm{http}: / /$ www.astro.princeton.edu/ rcool/hsred/hsred_reductions.html
} 
Table 2

MMT/Hectospec and WIYN/Hydra Fields

\begin{tabular}{|c|c|c|c|c|c|c|}
\hline Field & R.A. (J2000) & Decl. (J2000) & $\begin{array}{c}t_{\exp } \\
\text { (minutes) }\end{array}$ & $\begin{array}{c}\text { Observation Date } \\
\text { (UTC) }\end{array}$ & $\begin{array}{l}\text { Seeing } \\
\left({ }^{\prime \prime}\right)\end{array}$ & Airmass \\
\hline nep-hecto-1 & 175450 & +663847 & 100 & 2008 May 3 & $0.79^{*}$ & 1.22 \\
\hline nep-hecto-2 & 180429 & +655332 & 80 & 2008 Jun 2 & $2.18^{*}$ & 1.21 \\
\hline nep-hecto-3 & 175509 & +654928 & 80 & 2008 Sep 5 & $0.92^{*}$ & 1.57 \\
\hline nep-hecto-4 & 175929 & +671603 & 80 & 2008 Nov 17 & $1.34^{*}$ & 1.75 \\
\hline nep-hecto-5 & 180739 & +664156 & 80 & 2008 Nov 20 & $0.61^{*}$ & 2.05 \\
\hline NEP00 & 180010 & +663400 & 80 & 2008 Jun 27 & 0.7 & 1.35 \\
\hline NEP01 & 175629 & +662100 & 80 & 2008 Jun 27 & 0.7 & 1.30 \\
\hline NEP02 & 175409 & +655400 & 100 & 2008 Jun 27 & 0.8 & 1.30 \\
\hline NEP03 & 175151 & +661600 & 80 & 2008 Jun $28^{a}$ & 1.0 & 1.25 \\
\hline NEP04 & 175258 & +665800 & 80 & 2008 Jun 28 & 1.0 & 1.30 \\
\hline NEP05 & 175451 & +671000 & 60 & 2008 Jun $29^{b}$ & 1.2 & 1.25 \\
\hline NEP06 & 175928 & +671600 & 60 & 2008 Jun $30^{c}$ & 1.1 & 1.30 \\
\hline NEP07 & 180522 & +671000 & 60 & 2008 Jun 30 & 1.0 & 1.25 \\
\hline NEP08 & 180925 & +665900 & 60 & 2008 Jun 30 & 0.9 & 1.25 \\
\hline NEP09 & 180818 & +662000 & 60 & 2008 Jun 30 & 0.9 & 1.30 \\
\hline
\end{tabular}

Notes. * Seeing measured at the wavefront sensor. The MMT/Hectospec observations were performed in queue mode, thus the observing conditions were consistent from field to field.

a The guide star was relatively faint in the beginning of the night.

b Since the weather was getting cloudy, it was difficult to have the guide star. In the observatory site it rained after midnight.

$\mathrm{c}$ The wind was relatively strong in the beginning of the night, yet the overall weather condition was good.

spectra are spectrally typed by a comparison to the grid of Kurucz stellar models (Kurucz 1993), and the ratio between the observed spectral slope and that of best-fit model determines the sensitivity function of each fiber. The spectro-photometric zeropoint is obtained by combining the multi-band photometry of the observed F-type stars converted to SDSS ugriz photometry with their observed 1D spectra. After the flux calibration, each spectrum is de-reddened according to the Galactic dust maps of Schlegel et al. (1998) using the O'Donnell (1994) extinction curve. The Galactic extinction in NEP region is in the range of $E(B-V)=0.044-0.050$.

\subsection{WIYN/Hydra \\ 2.3.1. Observations}

We obtained optical spectra using the Hydra multi-object spectrograph on WIYN, the $3.5 \mathrm{~m}$ telescope at the Kitt Peak National Observatory. The field of view is approximately $1^{\circ}$ in diameter and the aperture size for each fiber is $2^{\prime \prime}$ in diameter. We used 98 red fibers feeding the bench spectrograph with a 316 lines $\mathrm{mm}^{-1}$ grating, yielding a spectral resolution of $5.7 \AA$ with a dispersion of $2.64 \AA$ pixel $^{-1}$ at blaze angle of $7^{\circ}$. The covered wavelength range is 4500-9000 $\AA$, yet the spectrum quality is very poor beyond $8000 \AA$ due to the presence of strong sky emission lines that were challenging to remove. The sensitivity is also low below $5000 \AA$.

The observations were performed on the nights of 2008 June 27-30. We observed ten fields over the entire NEP-Wide survey field, shown in Figure 3(b), where each spectroscopic setup is identified by a different color. The central coordinates of each configuration, the total on-source exposure time, and the observational dates are summarized in Table 2.

We used the WHYDRA software for spectroscopic target assignments. In each configuration, three to six fibers were assigned to spectro-photometric standard stars. F-type stars are selected as standard stars using the same criteria with those used for the Hectospec observations (see Section 2.2.1). Excluding broken fibers, fibers assigned to blank sky positions, and fibers assigned to standard stars, we obtained spectra of 60-70 targets in each configuration. In order to lock the object and sky fibers into correct source position, we used stars with $10<R<14$ from the Guide Star Catalog II (GSC 2.2; Lasker et al. 2008) as guide stars, but with coordinates transformed into the reference system used by the NEP survey. Ten to fifteen fibers per configuration were assigned to blank sky positions. The number of random sky positions was at least 10, to enable effective sky subtraction.

\subsubsection{Data Reduction}

We used IRAF to reduce the Hydra spectra. First, we performed pre-processing that includes overscan, bias and dark correction, flat-fielding, and trimming of the image. Flat-fielding was done using the average dome flats (halogen lamp) taken immediately before and after each observation in order to reduce fringing. Following the flat-fielding, cosmic rays were removed using L.A.Cosmic (van Dokkum 2001). After the preprocessing, we extracted 1D spectra using the Hydra reduction package DOHYDRA (Valdes 1995), designed for reduction and extraction of Hydra multi-fiber spectra. Once the 1D spectra were extracted and wavelength-calibrated with a comparison to $\mathrm{Cu}$-Ar lamp spectrum, skylines were subtracted. The master sky spectrum was produced by co-adding sky spectra of blank skies. Finally, the extracted spectra for different visits were combined using scombine to improve the $\mathrm{S} / \mathrm{N}$.

WIYN/Hydra spectra are also flux-calibrated by using the simultaneously observed F-type stars. We determined the spectral type of the observed F-type stars by comparing their ugriz photometry to a grid of Kurucz stellar models (Kurucz 1993), and using the best-fit model, we derived the sensitivity of each fiber as a function of wavelength. In some cases, the F-type stars observed thus used in the calibration are much bluer or redder than the color range spanned by the Kurucz stars, i.e., the observed photometry is not well matched to any of the 


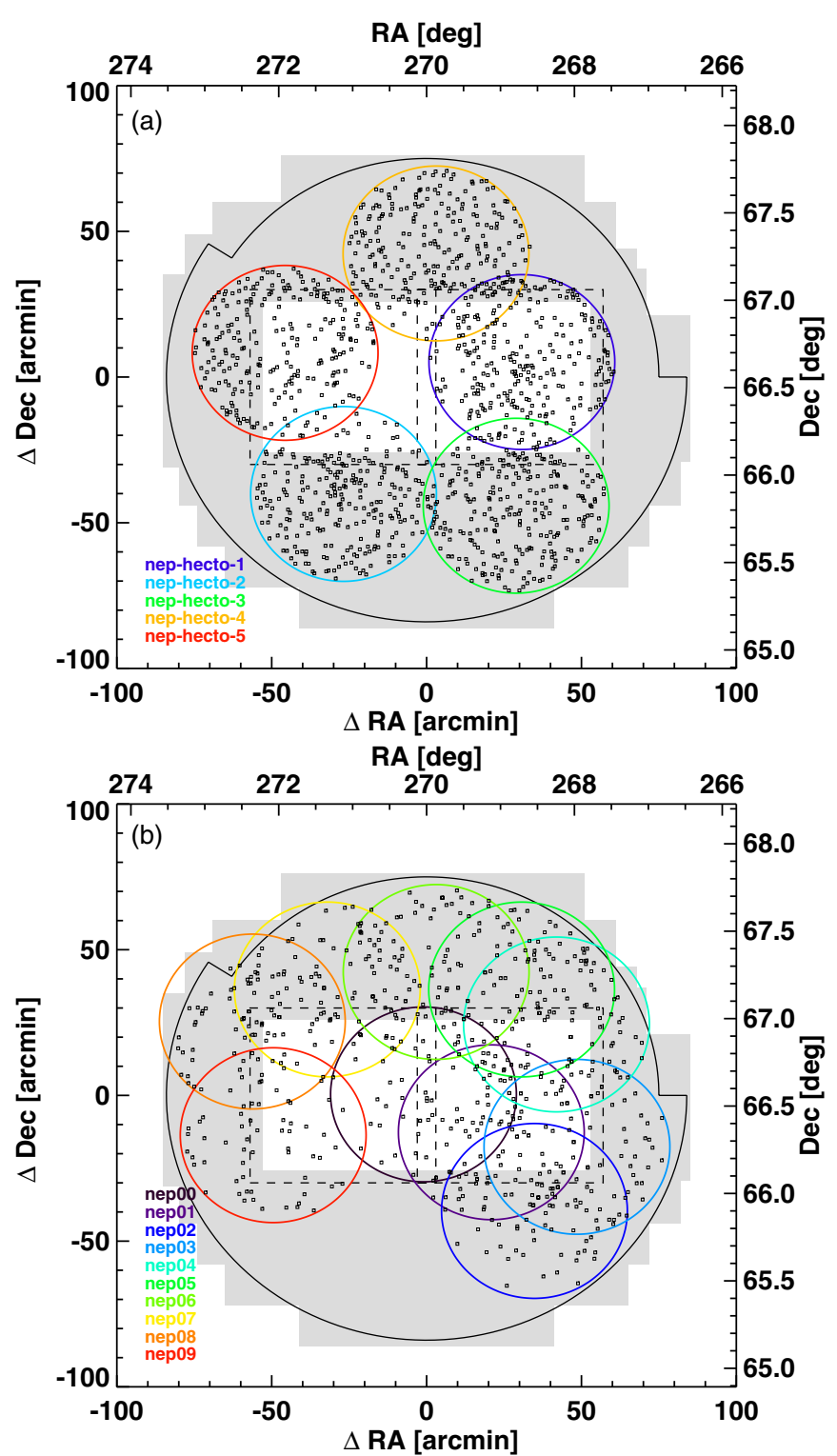

Figure 3. Follow-up spectroscopic observations of the NEP-Wide survey ((a) MMT/Hectospec fields; (b) WIYN/Hydra fields). The outermost solid circle represents the AKARI NEP-Wide IR (2-24 $\mu \mathrm{m})$ survey area $\left(5.4 \mathrm{deg}^{2}\right)$. The gray shaded region and dashed squares indicate the Maidanak $(B, R$, and $I$-band) survey area $\left(4.9 \mathrm{deg}^{2}\right)$ and the CFHT $(u, g, r, i$, and $z$-band) survey area $\left(2 \mathrm{deg}^{2}\right)$. Circles with different colors indicate different spectroscopic fields. Each small square corresponds to a spectroscopic target.

(A color version of this figure is available in the online journal.)

Kurucz stellar templates. This is either because our selection of the standard spectro-photometric stars was inappropriate or our conversion from BRI magnitudes to ugriz magnitudes lacked an additional color term. This remaining error was corrected by adding a multiplicative term to the sensitivity function, which is proportional to a power of the wavelength $\left(k \lambda^{\gamma}\right)$. The values for parameters $k$ and $\gamma$ are determined through the minimization of $\sum\left((R-I)_{\text {spectrum }}-(R-I)_{\text {observed }}\right)^{2}$. The spectro-photometric zeropoint, i.e., the normalization factor, is obtained through the comparison between the observed aperture magnitudes and the fiber magnitudes that reflect a convolution of the 1D spectrum with the filter response function.

Figures 4-6 illustrate the reliability of the flux calibration of the spectra by showing the comparison between the observed magnitudes (colors) and the estimated magnitudes (colors). The
$R$-, I-band magnitudes (Maidanak; Jeon et al. 2010) and $r$-, $i$-band magnitudes (CFHT; Hwang et al. 2007) are used for this comparison. The fiber ("spectroscopic") magnitudes were derived through the convolution of the observed flux-calibrated spectra and the filter response function. The observed ("photometric") magnitudes were measured using the aperture size of $3^{\prime \prime}$ that is roughly three times the FWHM (Jeon et al. 2010). This is slightly larger than the fiber size used in Hectospec $\left(1^{\prime \prime} .5\right)$ and Hydra ( $\left.2^{\prime \prime}\right)$ observations. Reducing the aperture size from $3^{\prime \prime}$ to $1^{\prime \prime} .5$ or $2^{\prime \prime}$ causes the observed magnitudes to be fainter by $0.1-0.4 \mathrm{mag}$, while the amount of magnitude change varies with filters. If the $1 .{ }^{\prime \prime} 5-2^{\prime \prime}$ diameter of a spectroscopic fiber is big enough to grasp the whole light from an object, the two (observed, estimated) magnitudes would be consistent with each other and there would be little scatter around $\Delta m=0$. However due to the various sizes of the spectroscopic targets, the difference between the FWHM of different observations, and the flux calibration uncertainties, we see a significant $\mathrm{rms}$ dispersion. The rms dispersions for $R$-band magnitudes (Figure 4), as well as I-band magnitudes (Figure 5), are in the range of $0.3-0.7 \mathrm{mag}$. The value is less in terms of $R-I$ colors: the rms dispersions for $R-I$ colors are in the range of $0.2-0.5 \mathrm{mag}$ (Figure 6).

The $R$ - or $I$-band magnitudes estimated from the fluxcalibrated spectra show a non-negligible scatter, implying that the flux calibration may contain a considerable amount of internal uncertainties. Both the continuum level and the emission line flux could have an uncertainty of $0.1-0.2$ dex. The $R$ - and I-bands are particularly the wavelengths at which the $\mathrm{H} \alpha$ emission line is redshifted, thus the typical uncertainty in $\mathrm{H} \alpha$ line flux would be of $0.1-0.2$ dex. We could not compare the calculated $B-R$ (or $g-r$ ) colors for Hydra spectra with the observed colors, since the $\mathrm{S} / \mathrm{N}$ of the spectrum drops significantly at wavelengths shorter than $5000 \AA$. But the Hectospec spectra show that the rms dispersion of the $B-R$ color difference ranges 0.4-0.9 mag. This implies that in the observed $\mathrm{H} \alpha / \mathrm{H} \beta$ line ratios, the uncertainty of $0.2-0.35$ dex would be included. We will discuss the effect of these internal uncertainties to our conclusions further in Section 5.3. The spectroscopically-derived properties, such as the SFR proportional to the emission-line flux, should be used with caution considering the large amount of flux calibration uncertainties reflected in the magnitude offset dispersion.

There exist several spectroscopic fields with especially large rms dispersion of magnitude offsets: among the Hectospec fields, the dispersion is larger in nep-hecto-1 and nep-hecto-5 fields compared to other fields. In the case of the Hydra fields, nep00 and nep01 fields are those with large dispersions. It is not clear what caused the scatter to be large in these fields. The quality of the selected F-type stars could be one of the reasons, considering that all of these fields are located near the center of the NEP field and share the spectrophotometric standards with non-typical optical color. Large airmass (nep-hecto-5; see Table 2) could also be responsible for the scatter.

\section{REDSHIFT IDENTIFICATION}

\subsection{Redshift Identification and Spectral Classification}

With the wavelength- and flux-calibrated spectra, we first measured the redshift of each object either through an automated pipeline in HSRED (Hectospec) or through a visual inspection of high $\mathrm{S} / \mathrm{N}$ emission lines (Hydra). Subsequently, the 

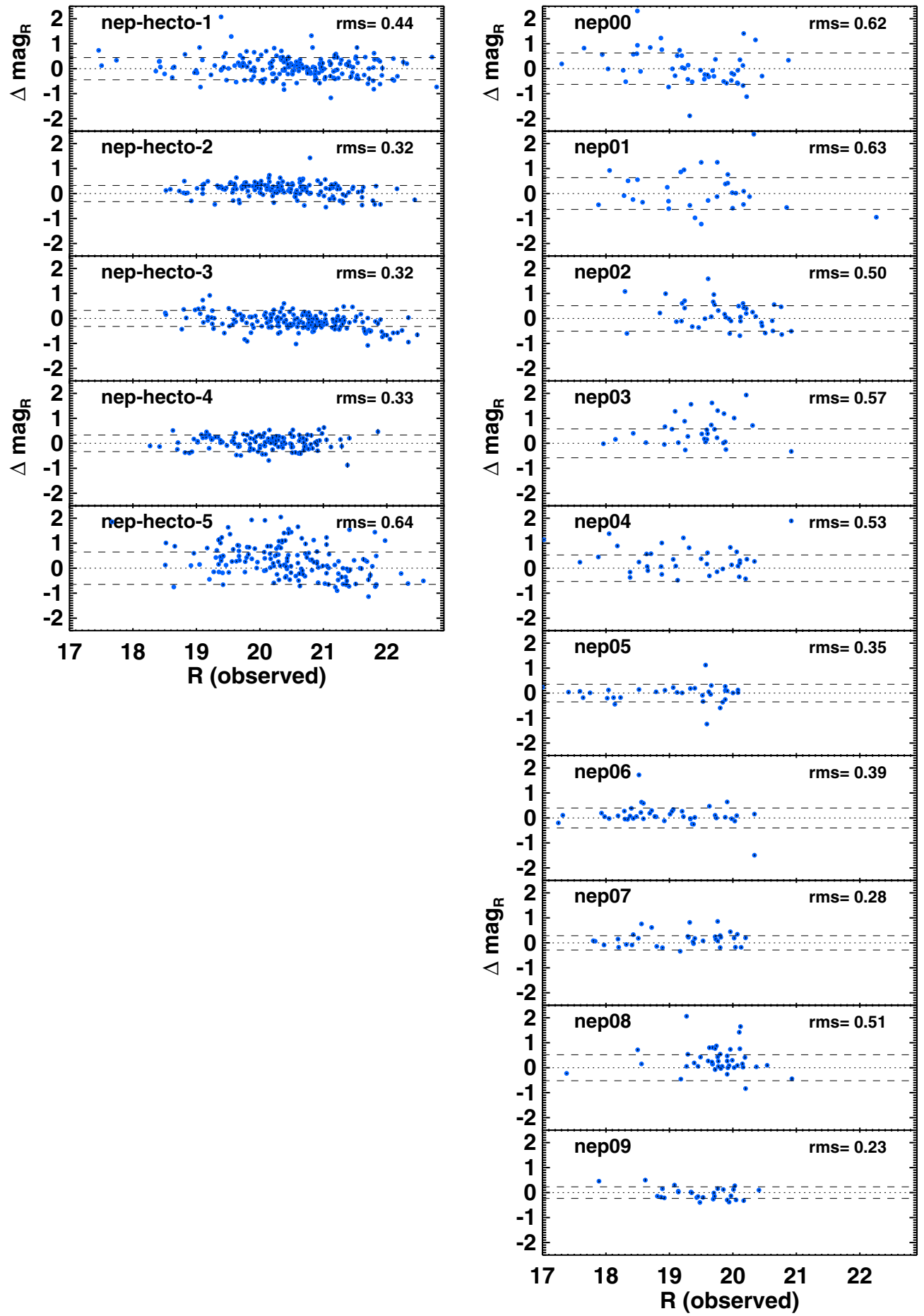

Figure 4. Comparison between the "photometric" aperture magnitudes $\left(R_{\text {phot }}\right)$ and the "spectroscopic" fiber magnitudes $\left(R_{\text {spec }}\right)$ in $R$-band. The $y$-axis indicates the difference between the aperture magnitudes and the fiber magnitudes: $\Delta m_{R}=R_{\text {spec }}-R_{\text {phot }}$. Dashed horizontal lines indicate rms dispersions for $\Delta m$, and dotted horizontal lines indicate $\Delta m=0$. The value of the rms dispersion in each field is specified in the top right. The more accurate the flux calibration of the spectrum is, the more consistent the two magnitudes should be.

(A color version of this figure is available in the online journal.)

"estimated" redshifts were individually validated by eye. Three authors (M. Im, J. Ko, and H. Shim) identified redshifts for all spectroscopically observed targets independently, by flagging the derived values according to the reliability and the significance of the spectral features used in the redshift measurement. Every object was assigned a quality flag ranging from 1 to 4: flag 4 for a secure redshift, flag 3 for an acceptable, almost good redshift, flag 2 for a questionable case, and flag 1 for cases where the redshift estimate is impossible. The flag system is similar with that used for the DEEP2 survey (e.g., Newman et al. 2012; Willmer et al. 2006).

The automated redshift identification pipeline for Hectospec data allows cross-correlation of the 1D spectrum with a series of galaxy, QSO, and stellar template spectra. ${ }^{11}$ The routine had a high success rate in the determination of redshifts: from the 1207 scientific targets observed by Hectospec, we found

\footnotetext{
11 http://www.sdss.org/dr7/algorithms/spectemplates/index.html
} 

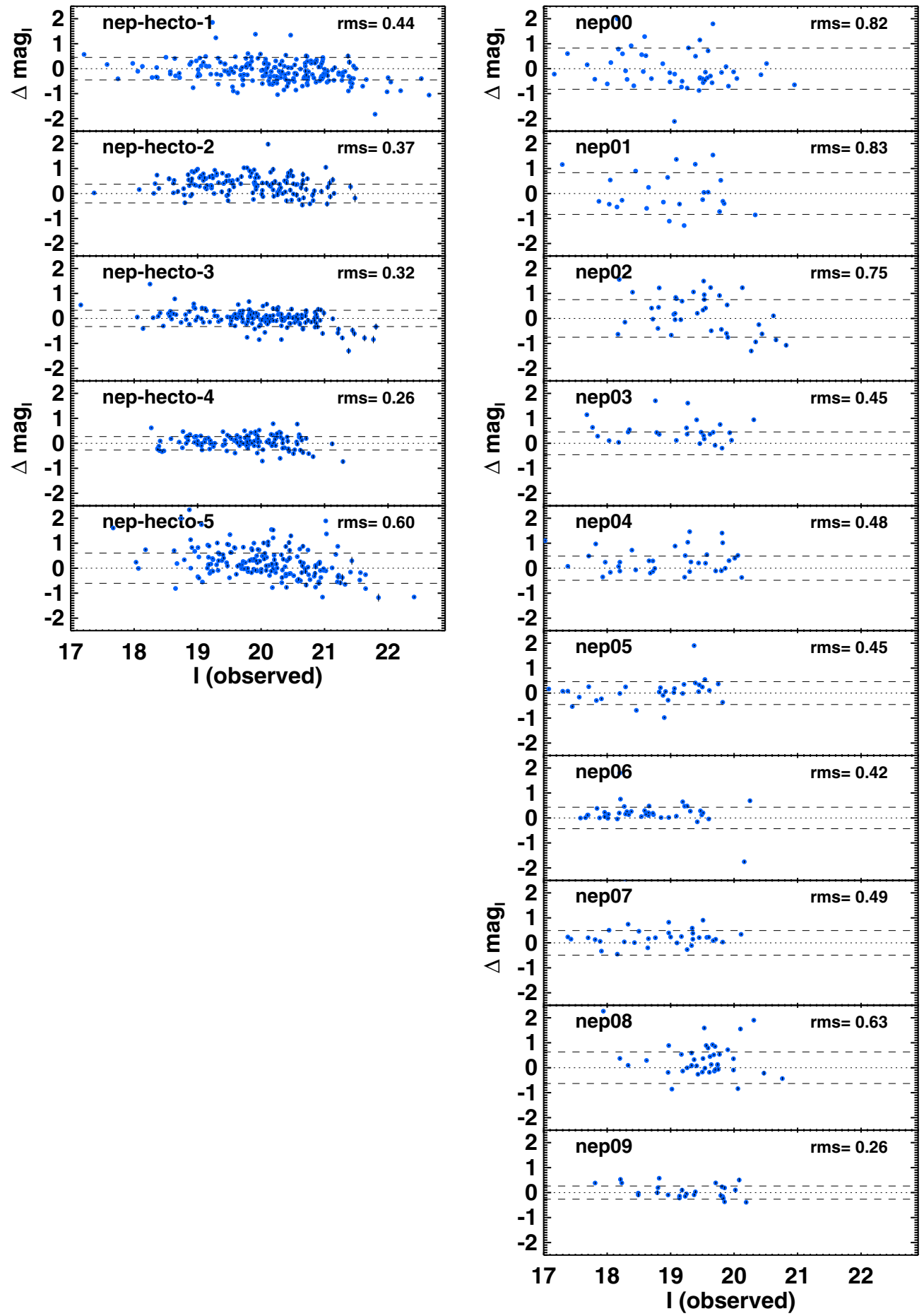

Figure 5. Comparison between the "photometric" aperture magnitudes $\left(I_{\text {phot }}\right)$ and the "spectroscopic" fiber magnitudes $\left(I_{\text {spec }}\right)$ in $I$-band. The $y$-axis shows the difference between the aperture magnitudes and the fiber magnitudes: $\Delta m_{I}=I_{\mathrm{spec}}-I_{\mathrm{phot}}$. Dashed horizontal lines indicate rms dispersions for $\Delta m$, and dotted horizontal line indicate $\Delta m=0$. The value of the rms dispersion in each field is specified in the top right. The more accurate the flux calibration of the spectrum is, the more consistent the two magnitudes should be.

(A color version of this figure is available in the online journal.)

that the redshifts derived from the pipeline returned doubtful values for only $52(0.5 \%)$ sources. Such suspicious redshift determination mostly happens for low $\mathrm{S} / \mathrm{N}$ sources identified to be high-redshift objects. For the majority of spectra with erroneous redshifts, we identified alternative redshifts by visual inspection using strong spectral features such as [O II], [O III], and $\mathrm{H} \alpha$. The central wavelength of an identified strong emission line was determined by Gaussian fitting, and this is compared to the vacuum wavelength of the emission line to calculate the redshift. However, in most cases where the redshift was not automatically derived, the lines are so weak, thus most of such sources are assigned a low quality flag-flags 1 or 2 .

For Hydra spectra, we measured redshifts by identifying high $\mathrm{S} / \mathrm{N}$ emission lines and/or multiple absorption lines adopting a similar method. The most frequently used emission lines are [O $\left.{ }_{\text {II }}\right] \lambda \lambda 3726,3729,\left[\mathrm{O}_{\text {III }}\right] \lambda \lambda 4959,5007$, and $\mathrm{H} \alpha+\left[\mathrm{N}_{\text {II }}\right]$ 

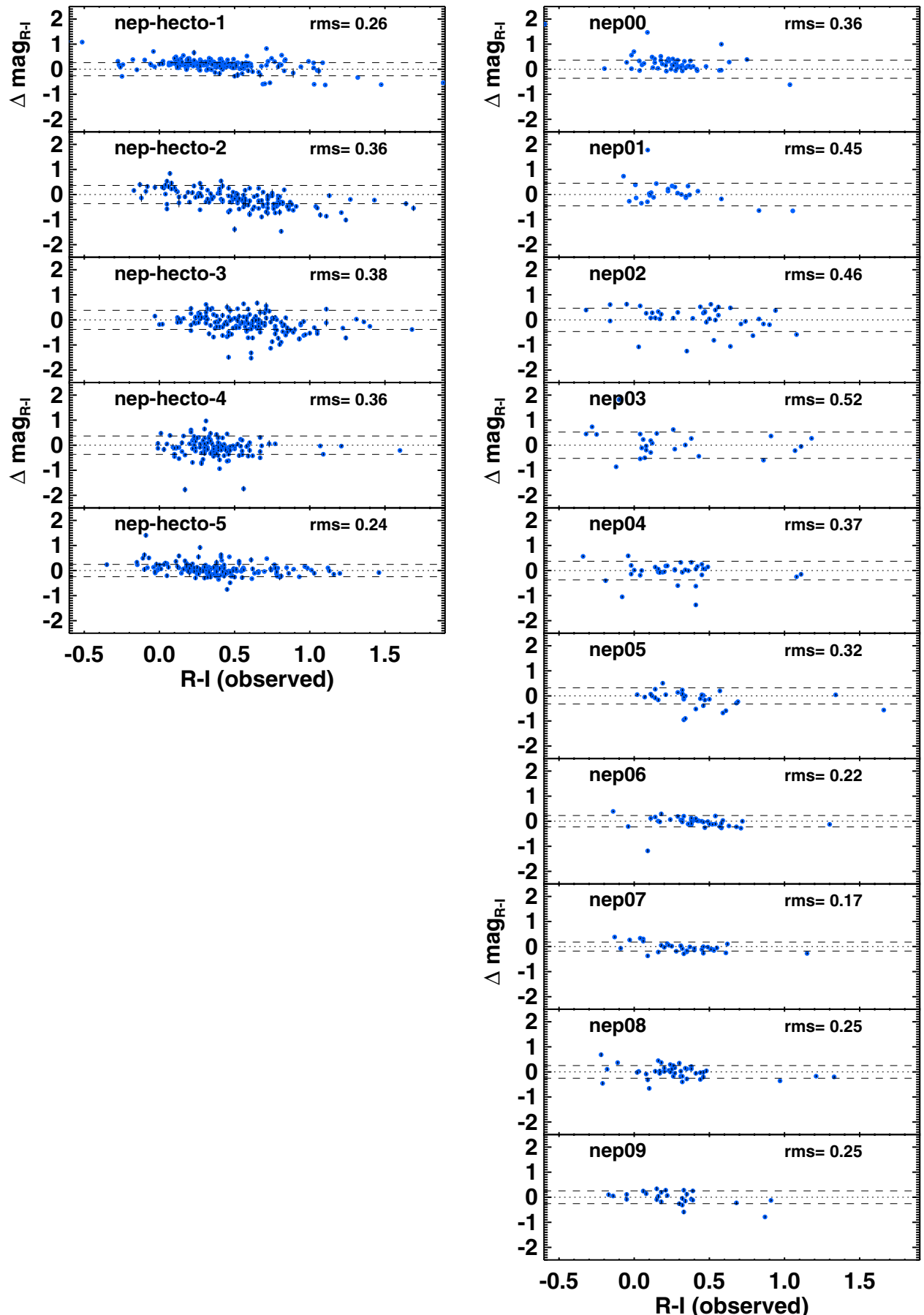

Figure 6. Comparison between the colors photometrically measured $\left((R-I)_{\mathrm{phot}}\right)$ and spectroscopically measured $\left((R-I)_{\mathrm{spec}}\right)$. The $y$-axis indicates the difference between the aperture colors and the fiber colors: $\Delta m_{(R-I)}=(R-I)_{\text {spec }}-(R-I)_{\text {phot }}$. Dashed horizontal lines indicate rms errors for $\Delta m$, and dotted horizontal lines indicate $\Delta m=0$. The value of the rms dispersion in each field is specified in the top right. The more accurate the flux calibration of the spectrum is, the more consistent the two colors should be.

(A color version of this figure is available in the online journal.)

complex. The other emission lines used for the redshift determination include $\mathrm{Ly} \alpha, \mathrm{C}$ IV $\lambda 1549$, He II $\lambda 1640, \mathrm{Mg}$ II $\lambda 2798, \mathrm{H} \gamma$, $\mathrm{H} \beta$, and [S II] $\lambda \lambda 6716,6731$. The absorption lines used in the redshift identification are the $\mathrm{Ca} \mathrm{H}$ and $\mathrm{K}$ doublet, $\mathrm{H} \delta, \mathrm{H} \gamma, \mathrm{H} \beta$, $\mathrm{Mg}$ absorption at $5175 \AA$, and $\mathrm{Na}$ at $5892 \AA$. We flagged the objects with at least two distinct spectral features as those with a secure redshift (quality flag 4). When the spectral features used in the redshift identification are not strong, a quality flag 3 is assigned to that object. If the redshift is determined using a single, low $\mathrm{S} / \mathrm{N}$ spectral feature or continuum break only, we assigned flag 2 to indicate that the redshift is highly unreliable. When the redshift identification is impossible, the object was given a quality flag 1 . All the three authors agreed on the secure redshifts. Flag 3 redshifts are mostly acceptable for general analysis. However, for faint or distant galaxies, the redshift can be ambiguous due to the weakness of spectral features. Therefore we recommend using quality flag 4 objects only in an analysis which highly depends on the accuracy of the redshifts.

To verify our redshift determination, we ran XCSAO in the RVSAO package, which computes radial velocities by 
Table 3

Optical Redshifts Catalog over NEP-Wide Survey

\begin{tabular}{|c|c|c|c|c|c|c|c|c|c|}
\hline Name & R.A. & Decl. & $\begin{array}{c}R \\
(\mathrm{mag})\end{array}$ & Redshift & Flag & Field & Fiber ID & Class & Identifier \\
\hline NEP_J174818.6+655754.7 & 267.07778 & 65.96519 & 18.37 & -0.0042 & 4 & nep03 & 20 & STAR & abs \\
\hline NEP_J174906.2+660027.1 & 267.27597 & 66.00753 & 19.79 & 2.4779 & 4 & nep03 & 68 & TYPE1 & $\mathrm{em}$ \\
\hline NEP_J174910.2+655223.1 & 267.29263 & 65.87308 & 19.83 & 0.1899 & 4 & nep03 & 64 & GALAXY & em+abs \\
\hline NEP_J175000.8+670125.7 & 267.50372 & 67.02381 & 17.45 & 0.0894 & 3 & nep04 & 6 & GALAXY & em \\
\hline NEP_J175011.8+663826.4 & 267.54937 & 66.64069 & 20.03 & 0.2147 & 4 & nep-hecto-1 & 198 & GALAXY & em+abs \\
\hline NEP_J175011.9+664205.5 & 267.54959 & 66.70153 & 99.00 & 0.9525 & 4 & nep-hecto-1 & 191 & GALAXY & abs \\
\hline NEP_J175136.3+665507.9 & 267.90136 & 66.91888 & 21.06 & 0.3907 & 4 & nep-hecto-1 & 226 & GALAXY & em+abs \\
\hline NEP_J175138.2+664131.2 & 267.90939 & 66.69200 & 18.92 & 0.4399 & 2 & nep03 & 10 & UNKNOWN & abs \\
\hline NEP_J175318.6+670650.2 & 268.32788 & 67.11396 & 18.14 & 0.0784 & 4 & nep-hecto-1 & 248 & GALAXY & em \\
\hline NEP_J175410.6+672154.4 & 268.54440 & 67.36513 & 18.65 & 0.2474 & 4 & nep05 & 32 & GALAXY & em+abs \\
\hline NEP_J175438.9+664310.6 & 268.66232 & 66.71961 & 18.17 & 0.0798 & 4 & nep01 & 21 & GALAXY & em \\
\hline NEP_J175533.1+660658.5 & 268.88809 & 66.11626 & 19.14 & 0.1982 & 4 & nep-hecto-3 & 53 & TYPE2 & em \\
\hline NEP_J175536.9+670937.5 & 268.90390 & 67.16043 & 19.71 & 0.1429 & 4 & nep-hecto-4 & 32 & GALAXY & em \\
\hline NEP_J180118.7+672355.1 & 270.32833 & 67.39866 & 19.24 & 0.2214 & 4 & nep07 & 20 & GALAXY & em \\
\hline NEP_J180119.3+672130.0 & 270.33053 & 67.35836 & 17.66 & 0.0549 & 4 & nep06 & 77 & GALAXY & em \\
\hline
\end{tabular}

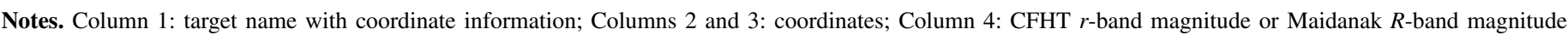

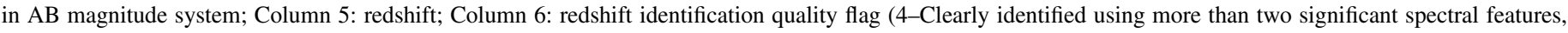

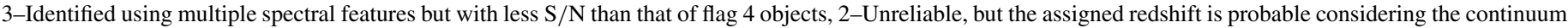

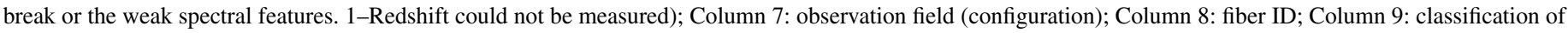

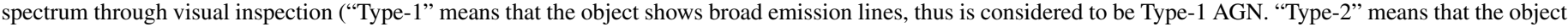

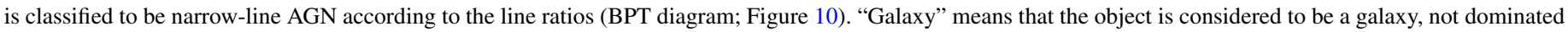

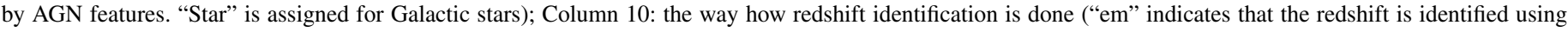
emission lines, "abs" using absorption lines, "em+abs" indicates using both).

(This table is available in its entirety in a machine-readable form in the online journal. A portion is shown here for guidance regarding its form and content.)

cross-correlating spectra against templates of known redshift (Kurtz et al. 1992). The test was done using 61 NEP supercluster member galaxies flagged as having secure redshifts, i.e., quality flag 4 . The radial velocities measured with XCSAO, the ones measured through the automated redshift determination routine used in Hectospec, and those measured using the visual inspection are all consistent with each other (Ko et al. 2012).

After the redshift identification, the spectra were classified into Type-1 AGNs, Type-2 AGNs, galaxies, stars, and unknown types again through a visual inspection. The classification was done separately by two authors (M. Karouzos and H. Shim), and only results where both classifications agreed with each other are presented in this paper. Objects with broad emission lines were classified as Type-1 AGNs. If the observed emission line ratios are consistent with that of AGNs and the emission lines lack broad components, the object was classified as Type-2 AGNs (see more detail in Section 4.2; Figure 10). In the case where the object was neither an AGN nor a star, it was classified as a galaxy unless the spectrum $\mathrm{S} / \mathrm{N}$ is too low for determination of spectral type.

Several examples of the reduced spectra from MMT/Hectospec and WIYN/Hydra observations are presented in Figure 7. The overlaid vertical lines indicate spectral features used in the redshift identification. The object name, the field ID, the fiber ID, and the redshift are specified as well as the object type. In Table 3, we present the redshift, the redshift flag, the method of the redshift identification (emission line, absorption line, or both) and the spectral classification of an object.

\subsection{Redshift Distribution of the Surveys}

The number of the spectra obtained by Hectospec observations is 1207, and the number of spectra obtained by Hydra observations is 651 , thus in total the number of spectra we have is 1858 . Considering multiple visits to one object, the net num- ber of objects spectroscopically observed is 1796. Among these, 1204 objects are assigned redshift quality flag 4, and 236 objects are assigned flag 3 . Therefore the overall success rate for redshift identification is $80 \%(1440 / 1796)$. However, the quality of the observational data strongly affects the redshift identification rate, thus the actual success rate is different for different observational fields. Figure 8 shows the redshift identification rate as a function of $R$-band magnitude. Although scatter exists between the fields, the success rate is larger than $50 \%$ at $R \sim 21-22 \mathrm{mag}$ for the Hectospec data. On the other hand, the redshift identification success rate drops below $50 \%$ at $R \sim 19$ mag for the Hydra data.

We show the redshift distribution in Figure 9, of 1645 objects with redshift quality flag equal or larger than 2 . About $90 \%$ of the objects have redshifts at $z<1$, with the redshift peak around $z \sim 0.2$. There exist two local redshift peaks at $z \sim 0.08$ and $z \sim 0.19$, reflecting the existence of galaxy clusters in the NEP-Wide field. The remaining $10 \%$ are distributed in the range of $1<z<4$. Objects with redshifts greater than 1 generally show AGN features in their optical spectra. The $R$-band magnitude limit of $<23$ mag roughly corresponds to a redshift limit of $z<0.6$.

\section{ANALYSIS OF EMISSION LINE FLUX}

\subsection{Line Flux Measurement}

From the flux-calibrated Hectospec and Hydra spectra, we measured fluxes of several significant emission lines ([O II $], \mathrm{H} \beta$, [O III], [O I $], \mathrm{H} \alpha+\left[\mathrm{N}_{\mathrm{II}}\right]$ complex, [S II $]$ ). Each flux-calibrated spectrum is first de-redshifted into the rest-frame using the spectroscopic redshift already identified above. Then the local continuum around the emission lines was determined through a first-order linear fit, and subtracted. On the continuumsubtracted spectrum, we applied a Gaussian profile fitting 


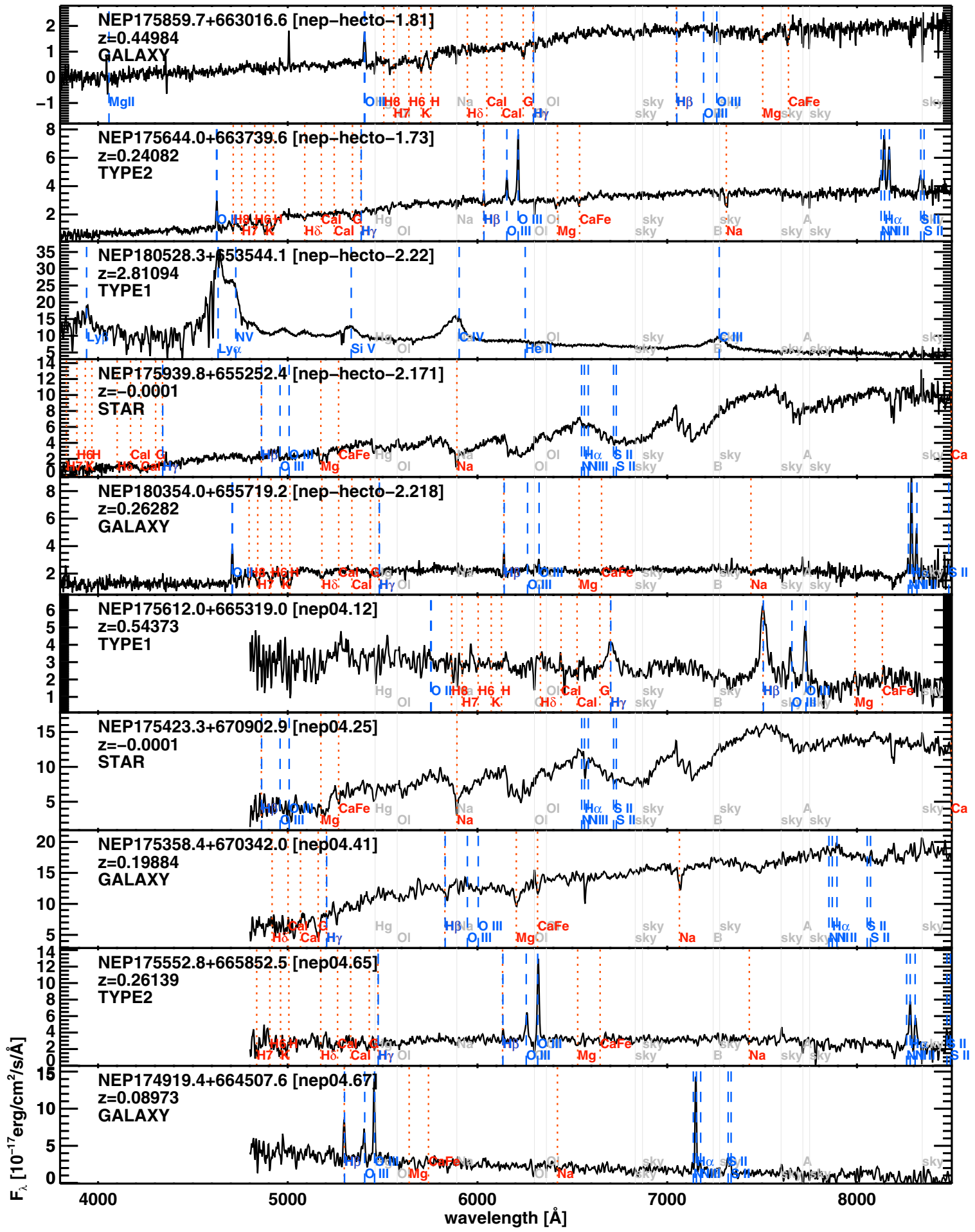

Figure 7. Example of WIYN/Hydra and MMT/Hectospec spectra classified as either galaxy, Type-1/Type-2 AGN, or star. Overlaid are sky (gray), absorption (red), and emission (blue) lines used in redshift identification. The unit for the $y$-axis is $10^{-17} \mathrm{erg} \mathrm{s}^{-1} \mathrm{~cm}^{-2} \AA^{-1}$. The object name, the field name, the fiber ID, and the redshift are specified.

(A color version of this figure is available in the online journal.)

using the MPFIT ${ }^{12}$ package based on the Levenberg-Marquardt method. The line center, the amplitude, and the FWHM were left as free parameters. In almost all cases, [O II] emission line doublet at 3726 and $3729 \AA$ is unresolved thus we used a single Gaussian to measure [O II] line flux. We used double Gaussians to fit the $\left[\mathrm{O}_{\mathrm{III}}\right] \lambda \lambda 4959,5007$ doublet and the $\left[\mathrm{S}_{\mathrm{II}}\right] \lambda \lambda 6716$,

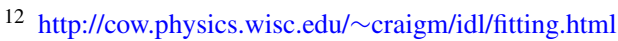

6731 doublet, and three Gaussians to fit the $\mathrm{H} \alpha+\left[\mathrm{N}_{\mathrm{II}}\right] \lambda \lambda 6548$, 6584 complex. For other lines, we used single Gaussian profiles.

When fitting Balmer emission lines $(\mathrm{H} \beta$ and $\mathrm{H} \alpha$ ), we also considered the stellar absorption in the determination of the local continuum since otherwise the nebular emission line fluxes can be underestimated because of the contribution from the underlying stellar absorption. We modeled the stellar absorption as a Gaussian with a broad FWHM and a negative amplitude. 

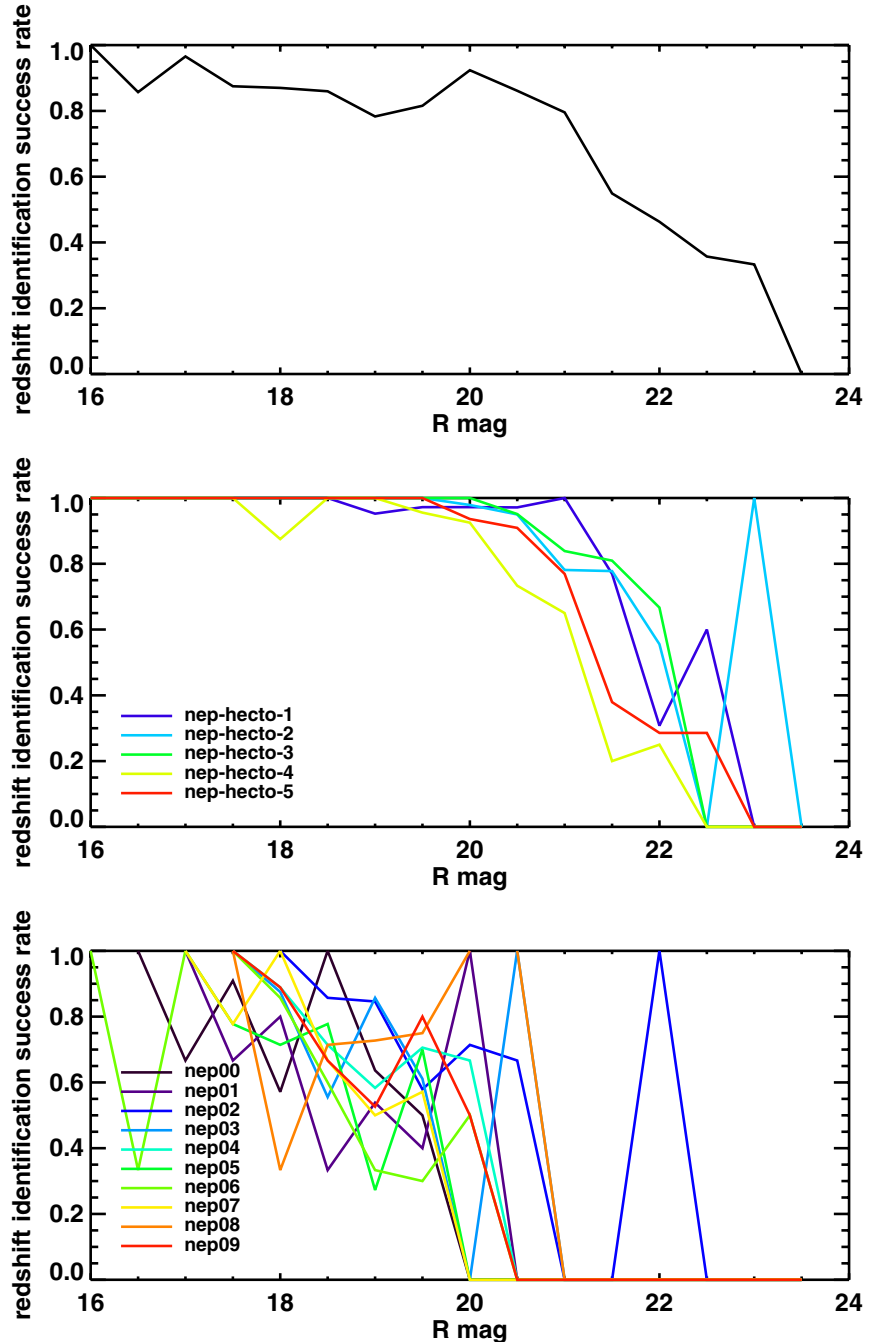

Figure 8. The success rate in the redshift identification as a function of $R$-band magnitude. Redshift success rate is defined as the number of sources with secure redshift (quality flags 3 and 4 ) divided by the number of total sources observed. The redshift identification success rate depends on overall data quality that reflects observational condition. For the Hectospec data, the redshift identification success rate drops below 50\% at $R=21-22$ mag for different fields. On the other hand, the rate drops more quickly (at $R \sim 18.5-21 \mathrm{mag}$ ) for Hydra data. Overall, the redshift identification is mostly successful at $R<21$ mag (top panel).

(A color version of this figure is available in the online journal.)

Generally the stellar absorption was not very strong; however, for some extreme cases of $\mathrm{H} \beta$, the existence of stellar absorption produced a flux change of up to $\sim 30 \%$.

The derived line fluxes are presented in Table 4 . The values in Table 4 are not corrected for the internal reddening of galaxies. As discussed earlier in Section 2.3.2, the uncertainties in the derived line fluxes are in the range $0.1-0.25$ dex. The uncertainties in the line ratios are lower if the lines fall in the wavelength range of the $R$ and $I$ band, but if the lines are located on $B$-band wavelengths, the line ratio uncertainties increase by a factor of $\sim 2$

\subsection{Diagnostic Line Ratios}

Emission line ratios, such as $[\mathrm{O} \mathrm{III}] / \mathrm{H} \beta$ and $[\mathrm{N}$ II $] / \mathrm{H} \alpha$, are effective indicators for separating populations with different ionizing sources (e.g., Baldwin et al. 1981; Kewley et al. 2001; Kauffmann et al. 2003). We present Baldwin-Phillips-Terlevich

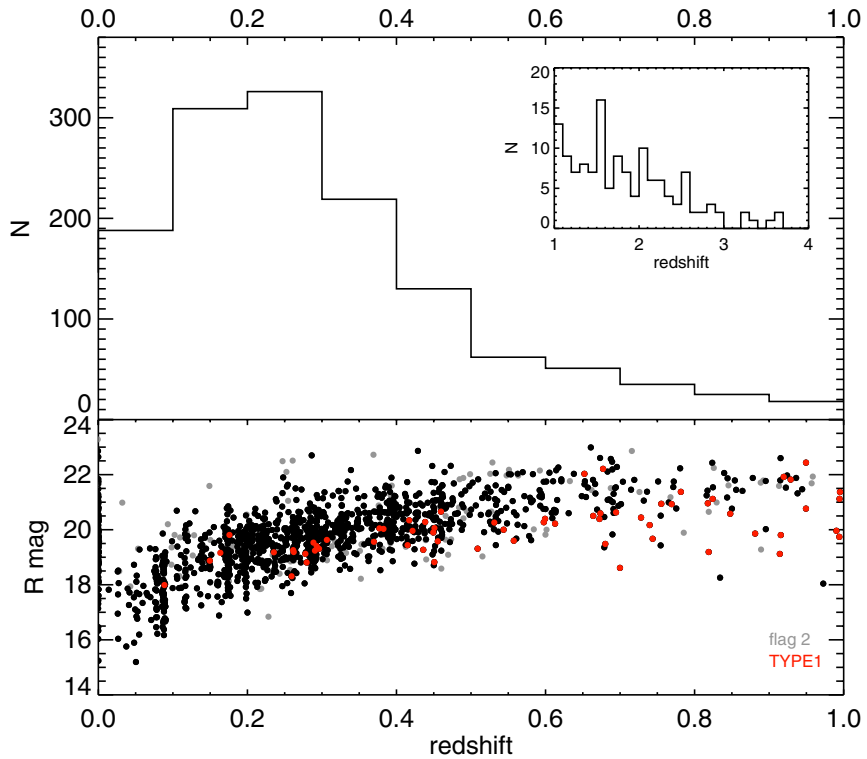

Figure 9. Redshift distribution of 1645 objects (with redshift quality flag equal or larger than 2). $90 \%$ of the objects (1499) have redshift less than 1, and the remaining $10 \%$ are considered to be at $z \gtrsim 1$ (see the inset plot). The magnitude limit of $R \sim 21 \mathrm{mag}$, at which redshift identification is mostly complete (see Figure 8), corresponds to the redshift of $z \sim 0$.6. Flag 2 objects (whose redshifts are questionable) are marked as gray points, and the objects classified as Type-1 AGN are marked as red points.

(A color version of this figure is available in the online journal.)

(BPT) diagrams for spectroscopic sources in Figure 10 to classify sources into star-forming (H II) galaxies, starburst-AGN composites (Comp), and AGNs.

First, objects under the extreme starburst model line (Kewley et al. 2001; dashed line in the leftmost panel of Figure 10$)$ in the $[\mathrm{O}$ III $] / \mathrm{H} \beta$ versus $[\mathrm{N} \mathrm{II}] / \mathrm{H} \alpha$ plot are classified as star-forming galaxies. According to the plot, the majority of the spectroscopically observed IR sources with emission lines are star-forming systems. The solid line in every panel indicates the criterion that divides AGN-dominated galaxies (upper right) and star-formation-dominated galaxies (lower left) as suggested by Kauffmann et al. (2003). The dotted lines show the range spanned by the Kauffmann et al. (2003) criteria considering the uncertainty included in line flux measurement. Sources above the dotted line are newly identified as narrow-line AGNs. In between the two, objects are described as a composite of starforming systems and AGNs yet these composite systems are not separately specified in the final table. In total, we were able to identify 8 additional narrow-line (Type-2) AGNs in addition to the 198 visually classified broad-line (Type-1) AGNs. These classifications are included in Table 3.

The dot-dashed line in the $[\mathrm{O} \mathrm{III}] / \mathrm{H} \beta$ versus $\left[\mathrm{S}_{\mathrm{II}}\right] / \mathrm{H} \alpha$ and $[\mathrm{O} \mathrm{I}] / \mathrm{H} \alpha$ panels is a divider for Seyfert 2 galaxies and the lowionization narrow emission-line region (LINER) introduced by Kewley et al. (2006). However, we do not attempt to distinguish whether the identified Type-2 AGNs are Seyferts or LINERs considering the uncertainty in the line flux ratio measurements.

\section{STAR FORMATION RATES OF GALAXIES}

\subsection{H $\alpha$ and [O II] Star Formation Rates}

First, we used $\mathrm{H} \alpha$ line fluxes for the estimation of SFRs of galaxies. To convert $\mathrm{H} \alpha$ fluxes into total SFRs, we used the 


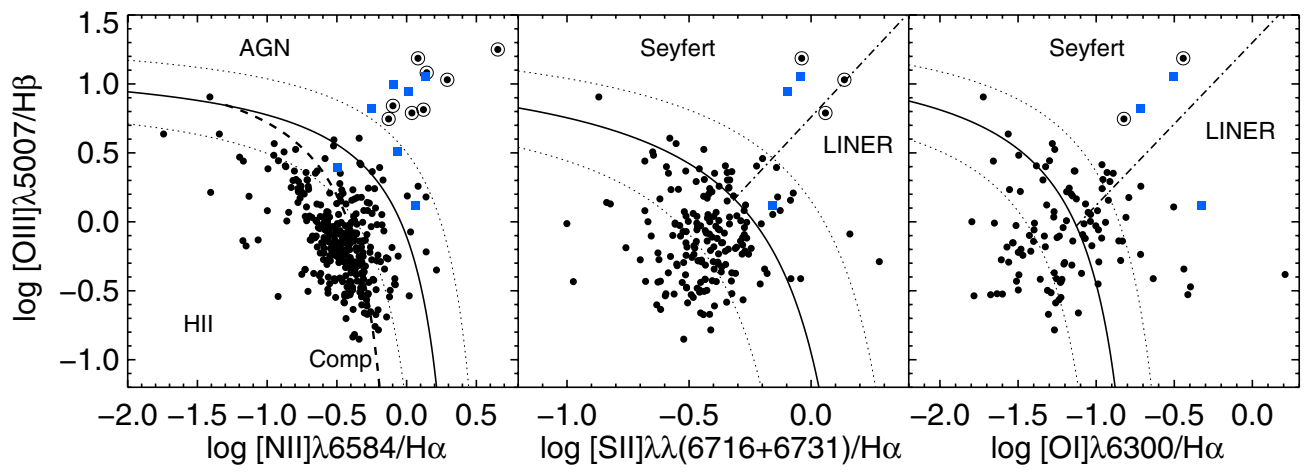

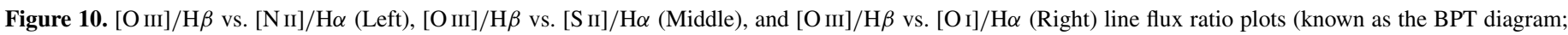
Baldwin et al. 1981) for our spectroscopic sample. The filled circles are galaxies with emission lines $(\mathrm{H} \beta,[\mathrm{O}$ III] $\lambda 5007,[\mathrm{~N}$ II] $\lambda 6584, \mathrm{H} \alpha)$ detected at $\mathrm{S} / \mathrm{N}$ larger than 3 , and the blue filled squares are Type-1 AGNs with broad emission lines. The double-circled objects are Type-2 AGNs identified through this BPT diagram analysis. (A color version of this figure is available in the online journal.)

Table 4

Line Flux Measurements for Selected Emission Lines

\begin{tabular}{|c|c|c|c|c|c|c|c|c|c|c|c|}
\hline Name & $\begin{array}{c}R \\
\text { (mag) }\end{array}$ & $\begin{array}{l}f([\mathrm{O} \mathrm{II}]) \\
(3727 \AA)\end{array}$ & $\begin{array}{c}f(\mathrm{H} \beta) \\
(4861 \AA)\end{array}$ & $\begin{array}{l}f([\mathrm{O} \text { III }]) \\
(4959 \AA)\end{array}$ & $\begin{array}{l}f([\mathrm{O} \text { III }]) \\
(5007 \AA)\end{array}$ & $\begin{array}{c}f([\mathrm{O} \mathrm{I}]) \\
(6300 \AA)\end{array}$ & $\begin{array}{l}f([\mathrm{~N} \mathrm{II}]) \\
(6548 \AA)\end{array}$ & $\begin{array}{c}f(\mathrm{H} \alpha) \\
(6563 \AA)\end{array}$ & $\begin{array}{l}f([\mathrm{~N} \mathrm{II}]) \\
(6584 \AA)\end{array}$ & $\begin{array}{c}f([\mathrm{~S} \text { II }]) \\
(6716 \AA)\end{array}$ & $\begin{array}{c}f([\mathrm{~S} \mathrm{II}]) \\
(6731 \AA)\end{array}$ \\
\hline NEP_J174818.6+655754.7 & 18.37 & $\ldots$ & $\ldots$ & $\ldots$ & $\cdots$ & $\cdots$ & $\cdots$ & $\ldots$ & $\ldots$ & $\cdots$ & \\
\hline NEP_J174906.2+660027.1 & 19.79 & -999.00 & -999.00 & -999.00 & -999.00 & -999.00 & -999.00 & -999.00 & -999.00 & -999.00 & -999.00 \\
\hline NEP_J174910.2+655223.1 & 19.83 & $\ldots$ & 25.34 & 34.97 & 18.45 & $\ldots$ & 0.33 & 41.48 & 10.24 & $\ldots$ & $\ldots$ \\
\hline NEP_J175000.8+670125.7 & 17.45 & $\ldots$ & $\ldots$ & $\ldots$ & $\ldots$ & $\ldots$ & $\ldots$ & 26.31 & 20.98 & $\ldots$ & $\ldots$ \\
\hline NEP_J175011.8+663826.4 & 20.03 & 10.69 & $\ldots$ & $\ldots$ & $\ldots$ & $\ldots$ & 3.34 & 28.91 & 17.95 & $\ldots$ & $\ldots$ \\
\hline NEP_J175011.9+664205.5 & 99.00 & $\ldots$ & -999.00 & -999.00 & -999.00 & -999.00 & -999.00 & -999.00 & -999.00 & -999.00 & -999.00 \\
\hline NEP_J175136.3+665507.9 & 21.06 & 14.27 & 5.43 & 1.26 & 3.89 & $\ldots$ & $\ldots$ & $\ldots$ & $\ldots$ & -999.00 & -999.00 \\
\hline NEP_J175138.2+664131.2 & 18.92 & $\ldots$ & $\ldots$ & $\ldots$ & $\ldots$ & $\ldots$ & -999.00 & -999.00 & -999.00 & -999.00 & -999.00 \\
\hline NEP_J175318.6+670650.2 & 18.14 & 34.44 & 10.37 & 7.53 & 18.73 & 4.55 & 1.59 & 44.12 & 7.35 & 8.74 & 8.74 \\
\hline NEP_J175410.6+672154.4 & 18.65 & $\ldots$ & 27.80 & $\ldots$ & $\ldots$ & $\ldots$ & $\ldots$ & 128.28 & 70.47 & $\ldots$ & $\ldots$ \\
\hline NEP_J175438.9+664310.6 & 18.17 & $\ldots$ & $\ldots$ & $\ldots$ & $\ldots$ & $\ldots$ & $\ldots$ & 145.97 & 13.54 & 51.16 & 49.12 \\
\hline NEP_J175533.1+660658.5 & 19.14 & 49.14 & 11.97 & 64.13 & 189.78 & 16.71 & 34.59 & 48.68 & 58.92 & 19.64 & 26.14 \\
\hline NEP_J175536.9+670937.5 & 19.71 & 20.42 & 21.96 & $\ldots$ & $\ldots$ & 4.85 & 24.72 & 138.92 & 69.17 & 24.72 & 14.71 \\
\hline NEP_J180118.7+672355.1 & 19.24 & $\ldots$ & 14.75 & $\ldots$ & $\ldots$ & $\ldots$ & 5.41 & 39.66 & 17.20 & 13.76 & 7.57 \\
\hline NEP_J180119.3+672130.0 & 17.66 & 150.69 & $\ldots$ & $\ldots$ & $\ldots$ & $\ldots$ & 14.63 & 185.70 & 56.76 & 65.36 & 29.61 \\
\hline
\end{tabular}

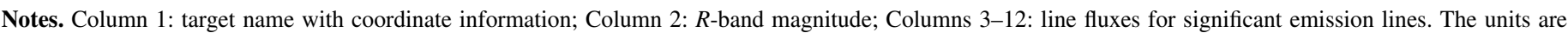
$10^{-17} \mathrm{erg} \mathrm{s}^{-1} \mathrm{~cm}^{-2}$. "-999" means that the line is redshifted out of the wavelengths covered by Hectospec/Hydra spectroscopic observation.

(This table is available in its entirety in a machine-readable form in the online journal. A portion is shown here for guidance regarding its form and content.)

following formula in Kennicutt (1998):

$$
\mathrm{SFR}_{\mathrm{H} \alpha}\left[M_{\odot} \mathrm{yr}^{-1}\right]=7.9 \times 10^{-42} L_{\mathrm{H} \alpha}\left[\mathrm{erg} \mathrm{s}^{-1}\right] .
$$

The conversion factor assumes a constant SFR and the Salpeter initial mass function (IMF; Salpeter 1955). The variation in the stellar IMF, age of the stellar population, and the star formation history would affect the derived SFR as well as the extinction (Kennicutt 1998). The use of $\mathrm{H} \alpha$ line fluxes as SFR indicator is limited to $z \lesssim 0.4$, since at higher redshifts, $\mathrm{H} \alpha$ is redshifted into the NIR wavelengths where the observed spectrum quality is poor due to the presence of strong $\mathrm{OH}$ skylines. Especially in our spectroscopic survey, $\sim 8500 \AA$ is the red limit of the spectrum thus we used $\mathrm{H} \alpha$ line as an SFR indicator only for galaxies at $z<0.3$. For galaxies at higher redshifts, we used [O II] line flux to estimate the SFR. The [O II]-SFR calibration is also adopted from Kennicutt (1998), and is based on the $[\mathrm{O}$ II $] / \mathrm{H} \alpha$ line ratios, meaning that the same assumptions on stellar population are applied; again the SFR derived using the following equation contains the internal uncertainties regarding the stellar IMF, age of the stellar population, and the star formation history of a galaxy:

$$
\operatorname{SFR}_{[\mathrm{O} \text { II }]}\left[M_{\odot} \mathrm{yr}^{-1}\right]=1.4 \times 10^{-41} L_{[\mathrm{O} \text { II }]}\left[\mathrm{erg} \mathrm{s}^{-1}\right] \text {. }
$$

\subsection{Infrared Star Formation Rates}

The SFR estimates from the optical emission lines ( $\mathrm{H} \alpha,[\mathrm{O}$ II $]$ ) are compared with the SFR estimates from the IR luminosity. We derived $L_{\mathrm{IR}}$ of each source (the integrated luminosity from 8 to $1000 \mu \mathrm{m}$ ) through the fitting of their IR SED. Galaxy templates with varying IR luminosities (Chary \& Elbaz 2001) are redshifted according to the spectroscopic redshift of an object, then the template that best describes the observed MIR photometric points $(N 2, N 3, N 4, S 7, S 9 W, S 11, L 15$ and $L 18 W$ ) is found by $\chi^{2}$ minimization. The MIR data are taken from the NEP-Wide catalog of Kim et al. (2012). Since the $L_{\mathrm{IR}}$ is basically extrapolated from the MIR data, the calculation assumes that the IR SEDs of star-forming galaxies remain the same at all epochs regardless of the $L_{\mathrm{IR}}$ and/or other factors including the properties of interstellar medium. Previous papers suggest that the $L_{\mathrm{IR}}$ derivation from the MIR is reliable within 


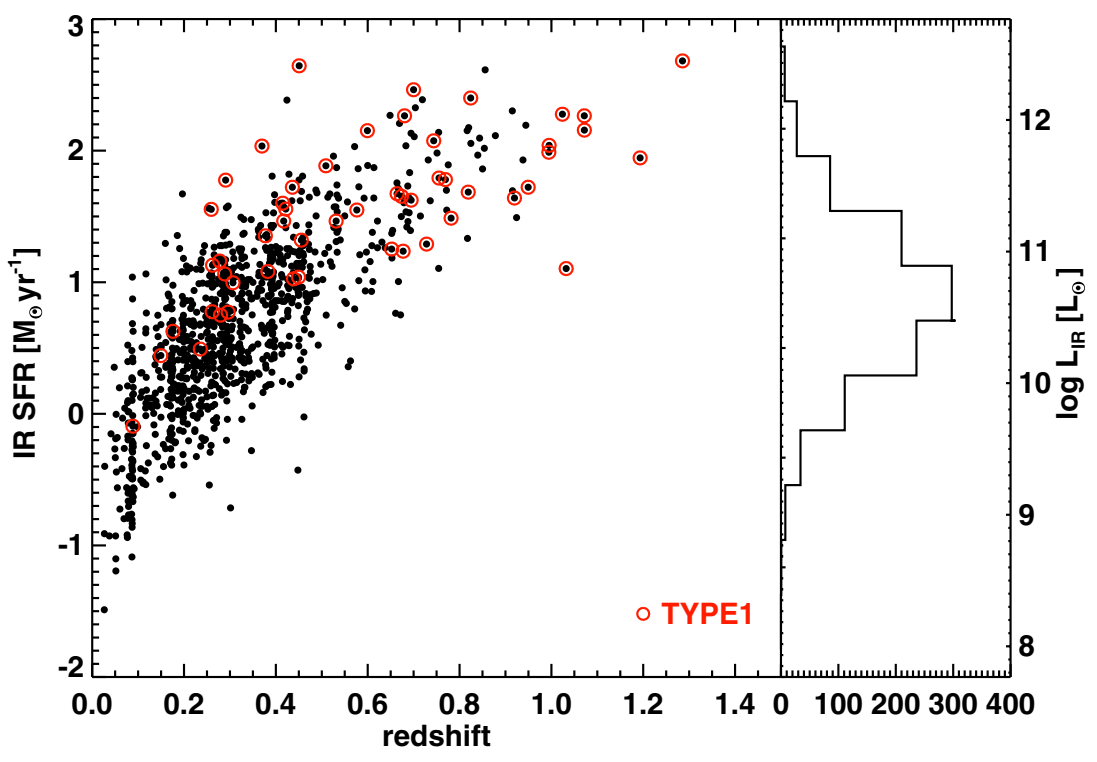

Figure 11. IR-derived SFR vs. redshift of the spectroscopic targets. The total IR luminosity $L_{\mathrm{IR}}$ ranges between $10^{9}$ and $10^{12} L_{\odot}$, yielding SFRs of $1-100 M_{\odot}$ yr ${ }^{-1}$. The Type-1 AGNs are plotted with red open circles, and their IR SFRs in this plot should be considered as upper limits. The objects at $z>1.5$ are omitted from the plot, since they are all Type-1 AGNs.

(A color version of this figure is available in the online journal.)

the uncertainty of $\sim 0.15$ dex at redshift below 1.3 (e.g., Elbaz et al. 2010, 2011). Considering the photometric errors in MIR bands and the IR SED model uncertainties, we estimate the uncertainty included in the derived $L_{\mathrm{IR}}$ of our targets to be a factor of 0.2 dex. With this $L_{\mathrm{IR}}$, SFR is estimated using the Kennicutt (1998) relation, which also is based on the constant SFR and the Salpeter IMF:

$$
\mathrm{SFR}_{\mathrm{IR}}\left[M_{\odot} \mathrm{yr}^{-1}\right]=4.5 \times 10^{-44} L_{\mathrm{IR}}\left[\mathrm{erg} \mathrm{s}^{-1}\right]
$$

As a result of $L_{\mathrm{IR}}$ derivation, we found 13 ultra-luminous IR galaxies (ULIRGs; i.e., $\left.L_{\mathrm{IR}}>10^{12} L_{\odot}\right)$ and 171 LIRGs $\left(L_{\mathrm{IR}}>\right.$ $10^{11} L_{\odot}$ ) in our spectroscopic sample. Seven of the ULIRGs and 30 of the LIRGs are AGNs, being half of the ULIRGs and $20 \%$ of the LIRGs respectively. ULIRGs and LIRGs consist $\sim 15 \%$ of the galaxies, and the remaining $85 \%$ are normal star-forming galaxies with a median $L_{\mathrm{IR}}$ of $2.5 \times 10^{10} L_{\odot}$. Figure 11 shows the $\mathrm{SFR}_{\mathrm{IR}}$ distribution of galaxies as a function of redshift, as well as the distribution of $L_{\mathrm{IR}}$.

\subsection{Extinction Correction}

A conventional method to estimate the internal extinction of a galaxy is to compare the observed and the intrinsic Balmer line ratios. For sources with both $\mathrm{H} \alpha$ and $\mathrm{H} \beta$ emission lines detected at $\mathrm{S} / \mathrm{N}>5$, we estimated the extinction by comparing the observed Balmer line ratio $\left(F_{o}^{\mathrm{H} \alpha} / F_{o}^{\mathrm{H} \beta}\right)$ with the intrinsic, unobscured line ratio $\left(F_{i}^{\mathrm{H} \alpha} / F_{i}^{\mathrm{H} \beta}\right)$ :

$$
E(B-V)_{\mathrm{gas}}=\frac{2.5}{[k(\mathrm{H} \alpha)-k(\mathrm{H} \beta)]} \log \left(\frac{F_{o}^{\mathrm{H} \alpha} / F_{o}^{\mathrm{H} \beta}}{F_{i}^{\mathrm{H} \alpha} / F_{i}^{\mathrm{H} \beta}}\right)
$$

The intrinsic Balmer line ratio $\left(F_{i}^{\mathrm{H} \alpha} / F_{i}^{\mathrm{H} \beta}\right)$ was set to 2.86, based on the assumptions of case $\mathrm{B}$ recombination and $T=$ $10,000 \mathrm{~K}$ (Osterbrock 1989). We used the reddening curve $k(\lambda)$ of Calzetti et al. (2000), which is a reddening curve for local starburst galaxies. The color excess in emission lines is known to be different from that calculated using the stellar continuum (e.g., Calzetti 2001) but the reason for the discrepancy is not clearly understood. In the following analysis, however, we consider $E(B-V)_{\text {gas }}$ as the color excess $E(B-V)$. The color excess is converted to the wavelength-dependent extinction and the $V$-band extinction using the following equations. Again, the reddening curve $k(\lambda)$ used is from Calzetti et al. (2000):

$$
\begin{gathered}
A(\lambda)=E(B-V) k(\lambda) \\
A_{V}=\frac{A(\lambda)}{k(\lambda)} k_{V} .
\end{gathered}
$$

The $A_{V}$ obtained this way is expressed as $A_{V}^{\mathrm{em}}$. Previous studies (Choi et al. 2006; Dominguez-Sanchez et al. 2012) suggested that there exists a positive correlation between the $V$-band extinction $A_{V}$ and the IR luminosity of galaxies at $L_{\mathrm{IR}}>10^{8} L_{\odot}$. To calculate the relationship between $A_{V}$ and $L_{\mathrm{IR}}$, these two studies used different approaches from each other. (See the dotted and the dashed lines in panels of Figure 12). In Choi et al. (2006), $A_{V}$ is calculated from the ratio between $\mathrm{SFR}_{\mathrm{IR}}$ and $\mathrm{SFR}_{\mathrm{H} \alpha}$, thus $A_{V}$ should be understood as $A_{V}^{\mathrm{IR}}$. In Dominguez-Sanchez et al. (2012), galaxies were divided into several bins using their $L_{\mathrm{IR}}$, and a composite spectrum was constructed for galaxies in each $L_{\mathrm{IR}}$ bin to increase the $\mathrm{S} / \mathrm{N}$ of the emission lines. Then $A_{V}$ was derived using the observed $\mathrm{H} \alpha / \mathrm{H} \beta$ ratio following the above Equations (4)-(6). In this case, $A_{V}$ is interpreted as $A_{V}^{\mathrm{em}}$.

We first assess the $A_{V}^{\mathrm{em}}$ versus $L_{\mathrm{IR}}$ correlation in comparison with the results from previous studies. Figure 12(a) illustrates the $A_{V}$ values calculated using the $\mathrm{H} \alpha / \mathrm{H} \beta$ line ratio as a function of $L_{\mathrm{IR}}$. Filled circles represent 146 objects at $0.02<z<0.3$ with both $\mathrm{H} \alpha$ and $\mathrm{H} \beta$ lines detected at $\mathrm{S} / \mathrm{N}$ greater than 5 . The Spearman's rank coefficient between the $A_{V}$ and $L_{\mathrm{IR}}$ is 0.17 , which corresponds to the probability of 0.03 that the plot is created through a random distribution of points. It is hard to say that there exists a significant correlation between the $L_{\mathrm{IR}}$ and $A_{V}^{\mathrm{em}}$. This is mainly because of the large uncertainties included in the line flux measurements which was expected from the large dispersion of the magnitude difference between the photometric and the spectroscopic data (Section 2.3.2). If we 

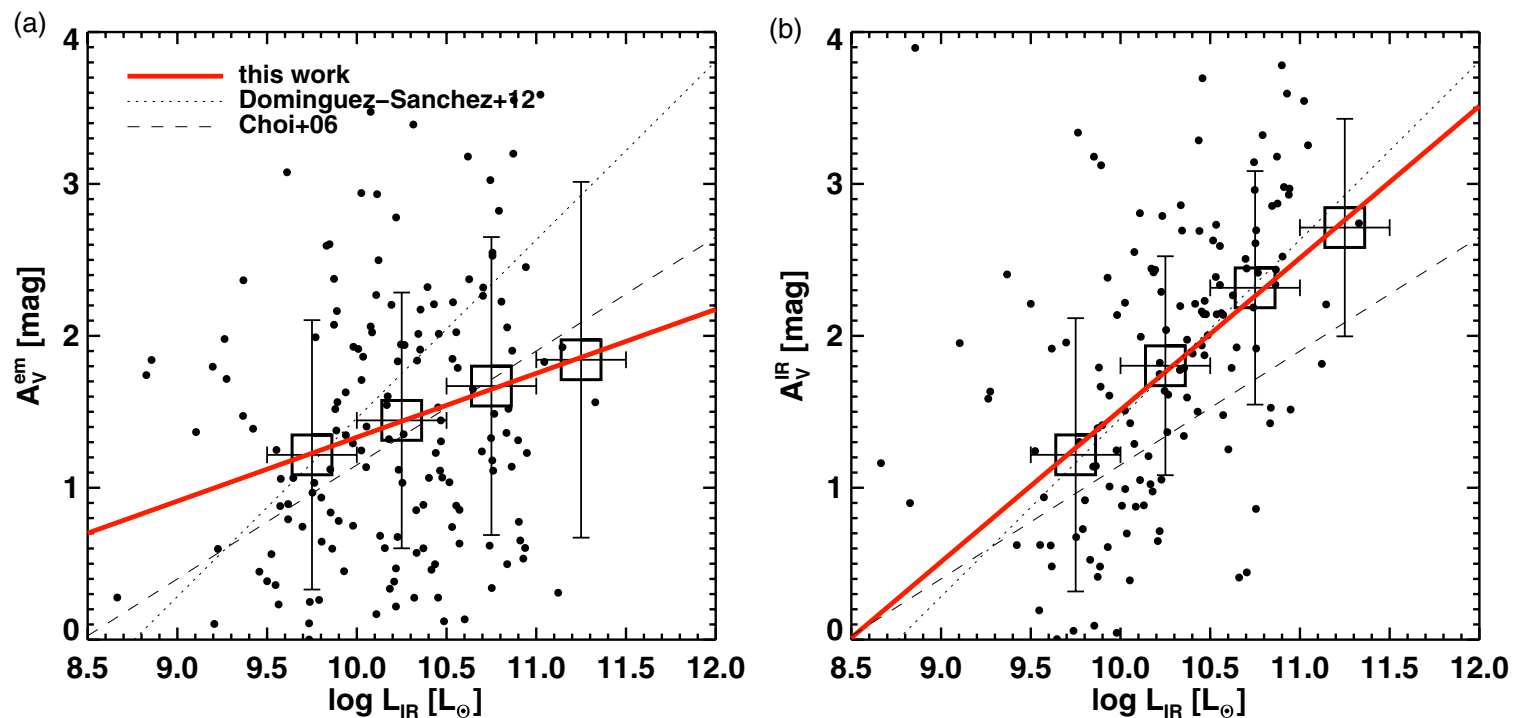

Figure 12. (a) Comparison between the $A_{V}$ from the observed $\mathrm{H} \alpha / \mathrm{H} \beta$ ratios and the IR luminosity. The squares indicate $\left\langle A_{V}\right\rangle$ for each $L_{\mathrm{IR}}$ bin. The red solid line is the best-fit for squares: $A_{V}^{\mathrm{em}}=-0.85+\left(0.23 \times \log L_{\mathrm{IR}}\right)$. Overplotted dotted and dashed lines show $A_{V}$ vs. $\log L_{\mathrm{IR}}$ relations presented in Dominguez-Sanchez et al. (2012; using emission line flux ratios) and Choi et al. (2006; using IR-optical SFR ratios), respectively. (b) Comparison between the $A_{V}$ calculated using the $\mathrm{SFR}_{\mathrm{H} \alpha} / \mathrm{SFR}_{\mathrm{IR}}$ ratios and the IR luminosity. The squares indicate $\left\langle A_{V}\right\rangle$ for each $L_{\mathrm{IR}}$ bin. The red solid line again indicates the linear fit for $A_{V}$ vs. $\log L_{\mathrm{IR}}$ using the squares: $A_{V}^{\mathrm{IR}}=-8.5+\left(1.0 \times \log L_{\mathrm{IR}}\right)$. The comparison of the two figures shows that the extinction correction based on the Balmer decrement underestimates the amount of the dust extinction at $L_{\mathrm{IR}} \gtrsim 10^{10} L_{\odot}$ and the trend is stronger at the higher $L_{\mathrm{IR}}$.

(A color version of this figure is available in the online journal.)

use the median of $A_{V}^{\mathrm{em}}$ of galaxies in the same $\log L_{\mathrm{IR}}$ bin (open squares) instead, over the $L_{\mathrm{IR}}$ range of $9.5<\log L_{\mathrm{IR}}\left(L_{\odot}\right)<12$, we obtain a linear relation of $A_{V}^{\mathrm{em}}=-0.85+0.23 \times \log L_{\mathrm{IR}}$ with an rms scatter of $0.89 \mathrm{mag}$. The scatter is still big, however we can use this relation as a proxy to estimate the amount of Balmer decrement from $L_{\mathrm{IR}}$ when the $\mathrm{H} \beta$ line is not present. The correlation is, if present, much weaker than that derived from the previous works (dashed and dotted line; Choi et al. 2006; Dominguez-Sanchez et al. 2012) as well as the slope of the correlation being flatter (compared to $0.75,1.17$ in Choi et al. 2006, Dominguez-Sanchez et al. 2012 respectively).

Another way to estimate the amount of extinction is to compare the IR-derived SFR with the optical or UV-derived SFR since IR luminosity samples the re-processed light from young or intermediate-age stars. Therefore, the ratio between $\mathrm{SFR}_{\mathrm{IR}}$ and $\mathrm{SFR}_{\text {opt }}$ (or SFR $\mathrm{UV}_{\mathrm{UV}}$ ) can serve as a measure of the amount of extinction. The extinction at a wavelength $\lambda$ is expressed as the following equation:

$$
A(\lambda)=2.5 \times \log \left(\mathrm{SFR}_{\mathrm{IR}} / \mathrm{SFR}_{\lambda}\right) .
$$

If the $A(\lambda)$ at $\mathrm{H} \alpha$ wavelength is converted to $A_{V}$ from the $\mathrm{SFR}_{\mathrm{IR}} / \mathrm{SFR}_{\mathrm{H} \alpha}$ ratio using the reddening curve (Calzetti et al. 2000), Equations (6) and (7), the $A_{V}$ value is considered to be $A_{V}^{\mathrm{IR}}$. Figure 12(b) shows the $A_{V}^{\mathrm{IR}}$ versus IR luminosity of 146 objects presented in Figure 12(a). The Spearman's rank coefficient is 0.52 , which gives the probability of $4.5 \times 10^{-11}$ that the plot arises from a random distribution. Thus compared to the case of $A_{V}^{\mathrm{em}}$ versus $\log L_{\mathrm{IR}}$, the relation for $A_{V}^{\mathrm{IR}}$ versus $\log L_{\mathrm{IR}}$ is more likely to exist. The best linear fit that describes the relation is $A_{V}=-8.5+1.0 \times \log L_{\mathrm{IR}}$.

It is challenging to conclude that there exists a positive correlation between the $L_{\mathrm{IR}}$ and the $A_{V}$ derived from two different methods. The correlation may exist; however, the large uncertainties included in the line flux measurement and the line flux ratio measurement may also have mimicked the correlation. Even if we use data with relatively $\operatorname{good} \mathrm{S} / \mathrm{N}$ in $\mathrm{H} \alpha$ and $\mathrm{H} \beta$ lines and small uncertainties in flux calibration only, the correlation remains weak unless we limit the allowed magnitude difference between the photometric and spectroscopic data to be less than $0.1 \mathrm{mag}$, which weed out almost all objects. Although the correlation between $L_{\mathrm{IR}}$ and $A_{V}$ has a large scatter, there is a marked difference between $A_{V}^{\mathrm{em}}$ and $A_{V}^{\mathrm{IR}}$ such that $A_{V}^{\mathrm{em}}$ is underestimated in comparison to $A_{V}^{\mathrm{IR}}$ and the difference is stronger when objects are more luminous in IR. This trend has already been noted in previous studies (e.g., Cardiel et al. 2003), and our result confirms the trend for a large number of IR sources.

Assuming that the $A_{V}$ versus $L_{\mathrm{IR}}$ correlation does exist, we compared the derived linear relation with that from the previous studies (Choi et al. 2006; Dominguez-Sanchez et al. 2012). Considering large flux calibration uncertainties of up to $0.35 \mathrm{dex}$, it is difficult to make a definitive statement on the comparison between our work and the other works. Given this caveat, we list two possible factors that may lead to the differences between our work and the previous works. First would be the redshift differences: our targets and targets of Dominguez-Sanchez et al. (2012) have a redshift range of $z<0.4$ while Choi et al. (2006) used a sample at a higher redshift with a median redshift of 0.7 . The effect of sampling a different redshift range is more significant at the luminous end, where the evolution of the IR SEDs of galaxies is considerable, thus our $A_{V}^{\mathrm{IR}}$ versus $L_{\mathrm{IR}}$ relation deviates from that of Choi et al.'s (2006) at large $L_{\mathrm{IR}}$. Secondly, the uncertainty in our flux calibration regarding the colors might weaken the $A_{V}^{\mathrm{em}}$ versus $L_{\mathrm{IR}}$ relation compared to that of Dominguez-Sanchez et al. (2012). The $R-I$ color differences between the calculated color and the observed color decrease as the observed $R-I$ increases (Figure 6), thus the $\mathrm{H} \alpha / \mathrm{H} \beta$ value gets lower when the galaxy is intrinsically red and higher when the galaxy is intrinsically blue. This may cause the $A_{V}^{\mathrm{em}}$ versus $L_{\mathrm{IR}}$ relation to be less steep, as appeared in Figure 12(a) in comparison with Dominguez-Sanchez et al. (2012). 

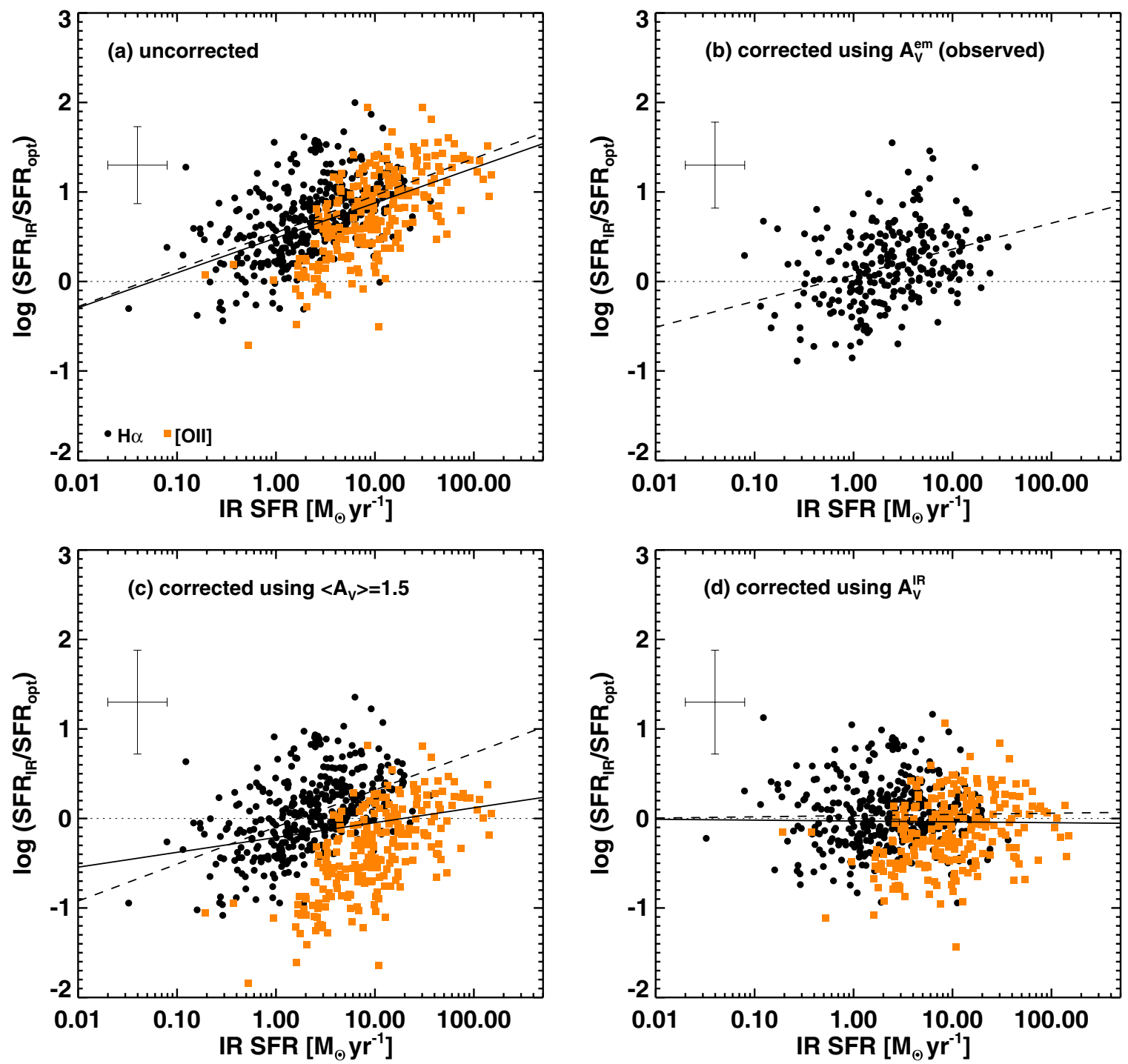

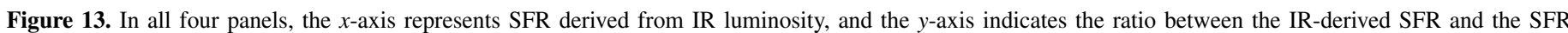

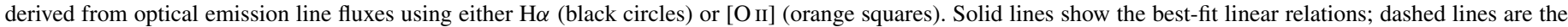

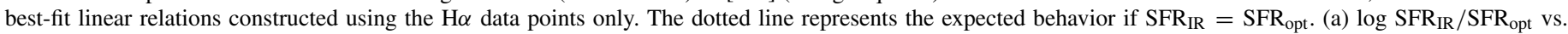

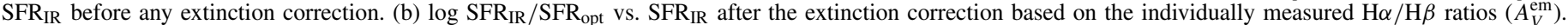

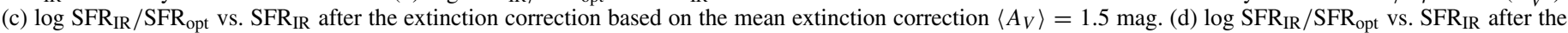
extinction correction based on $A_{V}^{\mathrm{IR}}$ vs. $\log L_{\mathrm{IR}}$ linear relation (red solid line in Figure 12(b)).

(A color version of this figure is available in the online journal.)

\subsection{Comparison between the Optical and IR-derived Star Formation Rates}

We applied the above extinction corrections to $\mathrm{H} \alpha$ or [O II] SFRs, and compared the extinction-corrected SFR with the IR-derived SFR. Figure 13(a) shows the ratio of $S_{F R}$ IR to $S F R_{o p t}$ as a function of SFR $\mathrm{IR}$ when no extinction correction is applied to the optical SFR. The difference between the IR- and the optSFR increases as the IR luminosity increases, suggesting that the extinction correction needed to correct the optically derived SFR increases as the $L_{\mathrm{IR}}$ of a galaxy increases. The trend is consistent whether the entire population is considered (solid line) or only the $\mathrm{H} \alpha$ points are considered (dashed line).

Figures 13(b)-(d) show the comparison between the SFRs derived using the $L_{\mathrm{IR}}$ and the $\mathrm{H} \alpha$ or [O II] line flux after the extinction correction. In Figure 13(b), we applied the extinction correction based on the individually detected $\mathrm{H} \alpha / \mathrm{H} \beta$ line ratio.
In Figure 13(c), we applied the constant extinction correction of $\left\langle A_{V}\right\rangle=1.5 \mathrm{mag}$. Figure 13(d) shows the result when the extinction correction based on the $A_{V}^{\mathrm{IR}}$ versus $\log L_{\mathrm{IR}}$ relation (solid line in Figure 12(b)) is applied.

Figure 13(b) shows that even if we apply the individual extinction correction using the observed Balmer decrement, the $\mathrm{SFR}_{\mathrm{IR}} / \mathrm{SFR}_{\text {opt }}$ ratios have large scatter of up to $\sim 0.5 \mathrm{dex}$ and the dependence of the SFR ratios on the $S_{F R}$ IR still remains. We already saw in Figure 6 that optically bluer (in $R-I$ color) galaxies are calibrated to be less blue and optically redder galaxies are calibrated to be less red. This effect in the color calibration propagates into the $\mathrm{H} \alpha / \mathrm{H} \beta$ ratios, thus the derived extinction correction for galaxies with initially less extinction would be overestimated and the derived extinction correction for highly extincted galaxies would be underestimated. Such overestimation and underestimation produced a remaining positive correlation between the $\mathrm{SFR}_{\mathrm{IR}} / \mathrm{SFR}_{\mathrm{opt}}$ and $\mathrm{SFR}$ IR even after the 
extinction correction. Not surprisingly, the extinction correction that best produces the $\mathrm{SFR}_{\mathrm{IR}}$ from the $\mathrm{SFR}_{\mathrm{opt}}$ is the best-fit $A_{V}^{\mathrm{IR}}$ versus $\log L_{\mathrm{IR}}$ relation (Figure 13(d)).

\section{SUMMARY}

We present MMT/Hectospec and WIYN/Hydra spectroscopic surveys of infrared sources over AKARI NEP-Wide Survey field. We targeted 1796 objects, among which spectroscopic redshift is clearly identified for 1440 objects (with flags 3 and 4). The success rate for redshift identification is higher than $80 \%$ at $R<21 \mathrm{mag}$, in case of Hectospec observations, though the quality of the spectra varies significantly according to the observation fields. The redshift catalog and line flux measurements are available as machine-readable table for this paper. The reduced spectra are also available on request.

The spectroscopic redshifts of $\sim 90 \%$ of the sources are distributed over the redshift range of $0.0<z<1.0$; the remaining $10 \%$ lie at $z>1.0$. Due to the target selection seeking high-redshift AGNs, most sources at $z>1.0$ are considered to be Type-1 AGNs with broad emission lines in addition to their special locations in the AKARI IRC color-color diagram. In addition to these bona fide AGNs, we identified narrowline AGNs based on the emission line diagnostics like the BPT diagram (Baldwin et al. 1981). Using [O III] $/ \mathrm{H} \beta$ versus $[\mathrm{NII}] / \mathrm{H} \alpha,[\mathrm{S} \mathrm{II}] / \mathrm{H} \alpha$ and $[\mathrm{OI}] / \mathrm{H} \alpha$ plots, eight sources are classified as Type-2 AGNs.

We compared the IR-derived SFR with the SFR derived from the optical emission line flux. The observed Balmer decrement $(\mathrm{H} \alpha / \mathrm{H} \beta)$ is still useful to estimate the extinction correction. However, due to the large uncertainty in the flux calibration, the extinction correction we measure using line ratios is underestimated for heavily extinguished systems and overestimated for relatively dust-free galaxies. We find that the $S F R_{I R} / S_{F R}$ opt ratio strongly depends on the $L_{\mathrm{IR}}$. Therefore, we estimated $A_{V}$ as a function of $L_{\mathrm{IR}}$, applied the extinction correction to the $\mathrm{H} \alpha$ or [O II] SFR, then compared the extinction-corrected optical SFR with the IR SFR. The two SFRs are consistent but with a large scatter of 0.38 dex.

This work was supported by the Creative Research Initiative program, No. 2010-0000712, of the Korea Research Foundation (KRF) funded by the Ministry of Education and Science and Technology of Korea. This research is based on the data obtained with MMT Observatory, a joint facility operated by the Smithsonian Institution and the University of Arizona; WIYN Observatory, a joint facility of the University of WisconsinMadison, Indiana University, Yale University, and the National Optical Astronomy Observatory operated by NOAO at KPNO; and $A K A R I$, a JAXA project with the participation of ESA. We thank $A K A R I$ NEP survey members for their inputs during the preparation of target lists.

\section{REFERENCES}

Baldwin, J. A., Phillips, M. M., \& Terlevich, R. 1981, PASP, 93, 5 Calzetti, D. 2001, PASP, 113, 1449

Calzetti, D., Armus, L., Bohlin, R. C., et al. 2000, ApJ, 533, 682 Cardiel, N., Elbaz, D., Schiavon, R. P., et al. 2003, ApJ, 584, 76

Chary, R., \& Elbaz, D. 2001, ApJ, 556, 562

Choi, P. I., Yan, L., Im, M., et al. 2006, ApJ, 637, 227

Doi, Y., Komugi, S., Kawada, M., et al. 2012, PKAS, 27, 111

Dominguez-Sanchez, H., Mignoli, M., Pozzi, F., et al. 2012, MNRAS, 426, 330

Elbaz, D., Dickinson, M., Hwang, H. S., et al. 2011, A\&A, 533, 119

Elbaz, D., Hwang, H. S., Magnelli, B., et al. 2010, A\&A, 518, 29

Fabricant, D. G., Kurtz, M. J., Geller, M. J., et al. 2008, PASP, 120, 1222

Goto, T., Hanami, H., Im, M., et al. 2008, PASJ, 60, 531

Goto, T., Takagi, T., Matsuhara, H., et al. 2010, A\&A, 514, 6

Hanami, H., Ishigaki, T., Fujishiro, T., et al. 2012, PASJ, 64, 70

Hwang, N., Lee, M.-G., Lee, H.-M., et al. 2007, ApJS, 172, 583

Im, M., Ko, J., Cho, Y., et al. 2010, JKAS, 43, 75

Ishihara, D., Onaka, T., Kataza, H., et al. 2010, A\&A, 514, 1

Jeon, Y., Im, M., Ibrahimov, M., et al. 2010, ApJS, 190, 166

Kauffmann, G., Heckman, T. M., Tremonti, C., et al. 2003, MNRAS, 346, 1055

Kennicutt, R. C. 1998, ARA\&A, 36, 189

Kewley, L. J., Dopita, M. A., Sutherland, R. S., Heisler, C. A., \& Trevena, J. 2001, ApJ, 556, 121

Kewley, L., Groves, B., Kauffmann, G., \& Heckman, T. 2006, MNRAS, 372,961

Kim, S. J., Lee, H. M., Matsuhara, H., et al. 2012, A\&A, 548, 29

Ko, J., Im, M., Lee, H. M., et al. 2012, ApJ, 745, 181

Kochanek, C. S., Eisenstein, D. J., Cool, R. J., et al. 2012, ApJS, 200, 8

Kurtz, M. J., Mink, D. J., Wyatt, W. F., et al. 1992, in ASP Conf. Ser. 25, Astronomical Data Analysis Software and Systems I, ed. D. M. Worrall, C. Biemesderfer, \& J. Barnes (San Francisco, CA: ASP), 432

Kurucz, R. L. 1993, PhST, T47, 110

Lasker, B. M., Lattanzi, M. G., McLean, B. J., et al. 2008, AJ, 136, 735

Lee, H. M., Im, M., Wada, T., et al. 2007, PASJ, 59, 529

Lee, H. M., Kim, S. J., Im, M., et al. 2009, PASJ, 61, 375

Marleau, F., Fadda, D., Appleton, P., et al. 2007, ApJ, 663, 218

Matsuhara, H., Wada, T., Matsuhara, S., et al. 2006, PASJ, 58, 673

Murakami, H., Baba, H., Barthel, P., et al. 2007, PASJ, 59, 369

Newman, J. A., Cooper, M. C., Davis, M., et al. 2012, arXiv:1203.3192

O’Donnell, J. E. 1994, ApJ, 422, 158

Ohyama, Y., Wada, T., Matsuhara, H., et al. 2009, in ASP Conf. Ser. 418, AKARI, A Light to Illuminate the Misty Universe, ed. T. Onaka, G. J. White, T. Nakagawa, \& I. Yamamura (San Francisco, CA: ASP), 329

Oke, J. B. 1974, ApJS, 27, 21

Onaka, T., Matsuhara, H., Wada, T., et al. 2007, PASJ, 59, 401

Osterbrock, D. E. 1989, Astrophysics of Gaseous Nebulae and Active Galactic Nuclei (Mill Valley, CA: University Science Books)

Owen, F., \& Morrison, G. E. 2009, ApJS, 182, 625

Oyabu, S., Ishihara, D., Malkan, M., et al. 2011, A\&A, 529, 122

Papovich, C., Cool, R., Eisenstein, D., et al. 2006, AJ, 132, 231

Salpeter, E. E. 1955, ApJ, 121, 161

Schlegel, D. J., Finkbeiner, D. P., \& Davis, M. 1998, ApJ, 500, 525

Shim, H., Im, M., Lee, H. M., et al. 2011, ApJ, 727, 14

Takagi, T., Ohyama, Y., Goto, T., et al. 2010, A\&A, 514, 5

Valdes, F. 1995, Guide to the HYDRA Reduction Task DOHYDRA, http://iraf.net/irafdocs/dohydra.pdf

van Dokkum, P. G. 2001, PASP, 113, 1420

White, G. J., Pearson, C., Braun, R., et al. 2010, A\&A, 517, 54

Willmer, C. N. A., Faber, S. M., Koo, D. C., et al. 2006, ApJ, 647, 853 